\title{
On the Practicality of Abstract Equation Systems*
}

\author{
Anne Mulkers Wim Simoens ${ }^{\dagger} \quad$ Gerda Janssens Maurice Bruynooghe \\ Department of Computer Science, K.U.Leuven, \\ Celestijnenlaan 200 A, 3001 Herverlee, Belgium, \\ \{anne,gerda,maurice\}@cs.kuleuven.ac.be wsi@sunbim.be \\ Tel. $++32(0) 16-3277$ 00, Fax. $++32(0) 16-327996$.
}

November 1994

\begin{abstract}
Data-flow analyses for mode and variable sharing information are of great interest in optimizing compilers for logic languages. After research at the foundational level and a study of alternative approximation domains, an extensive experimentation with various formalisms enables a better assessment of their potential value and may expose possible weaknesses and inadequacies. To that end a parametric combined data-flow analysis for logic programs based on the formalism of abstract equation systems is implemented and evaluated with respect to its accuracy and efficiency. The impact of each component of the domain on the precision and complexity of the analysis is measured. The experiments provide evidence that the domain generalizes several prior analyses and is suited as a basis for a flexible analysis tool. We report on the comparison with analyses such as Pat $(\mathcal{R})$, Sharing, Sharing+Free, and Sharing+Free+Linear.
\end{abstract}

\section{Introduction}

In [10], Codish et al. introduced a novel domain of abstract equation systems aimed at describing possible sharing and definite freeness of terms. A simple and intuitive abstract unification algorithm mimicking the classic Martelli-Montanari unification algorithm was presented. However, the algorithm was not very practical since it often produces a set of abstract equation systems. An attempt was made to remedy this in [4] where the abstract equation systems were augmented with a sharing component - expressing possible sharing between abstract terms using the set-sharing representation [23, 24] — and an annotation mapping - expressing freeness and linearity properties of the abstract terms. By extending the Martelli-Montanari unification algorithm with a few equivalence preserving rewrite rules, an abstract unification algorithm was formulated as a set of rewrite rules such that each rule mimics a small set of rule applications in the concrete system and a single solved form is computed. In further work, the approach has been substantially improved by integrating so-called mixed terms (compound terms having abstract terms as components), by defining the other operations needed by an application, and by proving

*This research has been funded in part by the National Fund for Scientific Research, Belgium, the ESPRIT project 5246 PRINCE, CEC, and by the GOA "Exploitation of Abstract Interpretation for Logic Programs", Belgium.

${ }^{\dagger}$ Present address: BIM n.v./s.a., Kwikstraat 4, B-3078 Everberg, Belgium. 
the operations correct $[5,6]$. The resulting system derives depth- $k$ structural information together with definite equality between (sub)terms [7, 25, 30,31]. The current paper presents a parameterized version of the domain of $[5,6]$ and reports on an implementation to illustrate the practicality.

The implementation of the abstract equation systems domain, $\mathcal{A} E q s(k, \mathcal{A})$, was designed as a tool for comparing several instantiations. First, if the depth $k$ is set to zero and the annotation mapping is a dummy mapping (into the annotation lattice $\mathcal{A}=\{a\}$, where $a$ means any term), the domain reduces to the basic set-sharing domain, Sharing, developed by Jacobs and Langen [23, 24, 34], which derives groundness information and has sharing information expressed at the level of program variables. Second, also using depth $k$ equal to zero but an annotation mapping based on the lattice of annotations $\mathcal{A}=\{f, a\}$, where $f$ means definitely free, $a$ means any term, and $f \leq a$, we simulate the Sharing+Free domain of Muthukumar and Hermenegildo [36], where the freeness information can be used to derive more accurate possible variable sharing information. If depth $k$ is non-zero in the cases above, we obtain analyses that can be considered as instantiations of the Pat $(\mathcal{R})$ domain of $[15,30,31]$ with $\mathcal{R}$ being the Sharing and Sharing+Free domain respectively. The Pat $(\mathcal{R})$ domain was developed to extend existing variable sharing and mode domains in an easy and modular way with depth-bounded structural and termequality information. Finally, we consider an annotation mapping based on the lattice of annotations $\mathcal{A}=\{f, l, a\}$, where $l$ means definitely linear and $f \leq l \leq a$. A term is linear if no variable occurs more than once in it. The linearity information can be used to further improve the precision of the basic set-sharing domain. The domain obtained is Sharing+Free+Linear, proposed by Bruynooghe and Codish in [4]. For depth $k$ equal to zero, the expressivity of the domain is comparable to the reduced product domain $\mathrm{P} \star \mathrm{SF}$ of [13], while for $\mathcal{A}=\{l, a\}$ it is comparable to the reduced product domain $\mathrm{P} \star \mathrm{S}$ of [13]. For depth $k>0$, definite structural information is derived and the sharing information is represented to a higher degree of accuracy than when expressed at the level of program variables; in this case, there is a relationship with the work of King and Soper [28]. Full specifications of the domain dependent operations and correctness proofs are in [6]. The latter report considered an even more elaborate annotation mapping, $\mathcal{A}=\{f, l, a, c, c l\}$, for deriving definite boundedness $(c$ or $c l)$ information. Alternative sets of annotations could be considered as studied by Getzinger [20].

The contribution of the work on abstract equation systems is twofold. First, it provides an interesting alternative for the $\operatorname{Pat}(\mathcal{R})$ domain of $[15,30,31]$ in that the basic operations are specifiable at a higher level and reuse well-known algorithms for unification, anti-unification, and a generalization of the concept of sharing groups [23,34] instead of the somewhat tricky operations of, e.g. [15]. Second, the implementation of the parametric domain enables experiments for comparing efficiency and precision aspects of various instantiations. They provide valuable information for choosing an appropriate candidate for eventual integration in a compiler. We observed a relatively small impact of definite structural (or type) information on the precision of variable sharing, freeness, and linearity information. However, structural information by itself is very useful data for an optimizing compiler. In order to control the complexity of the analysis, the requirements of the compiler should determine the amount of structural information that is maintained during the analysis.

Section 2 presents the domain of abstract equation systems. In Section 3, we discuss the precision and efficiency of different instantiations of the domain. Section 4 contains a discussion of related work and Section 5 some concluding remarks. 


\section{Abstract Equation Systems}

In this section, we first recall some basic definitions from [6] and then present the abstract unification algorithm which is the basic operation of the abstract domain. Other operations needed in a framework for abstract interpretation $[2,3,12,16,26,32]$, such as procedureentry and procedure-exit, and even builtin predicates such as arg $/ 3$ and $=\ldots / 2$, are special cases of abstract unification.

\subsection{Preliminaries}

By $A, B, C$, etc. we denote arbitrary concrete variables (Var denotes a denumerable set of concrete variables); abstract variables $@, @_{i}, @^{\prime}$, etc. (from the denumerable set AVar) are introduced to represent terms whose internal structure is hidden by abstraction (we assume that $A$ Var $\cap$ Var $=\emptyset$ and that @ is a distinguished symbol not used elsewhere as a function symbol); $t, t_{i}, \tau, \tau_{i}$, denote mixed terms $(\in \mathrm{MTerm})$, i.e., terms over $\vee a r \cup$ AVar. The set of terms over the set of concrete variables Var is denoted Term. The sets of concrete variables and abstract variables occurring in a syntactic expression $e$ are denoted by $\operatorname{Var}(e)$ and $A \operatorname{Var}(e)$, respectively. The power set of $S$ is denoted $\wp(S)$ and by $\doteq$ we denote syntactic identity.

An equation over a set $T$ of terms is an object of the form $t_{1}=t_{2}$ where $t_{1}, t_{2} \in T$. An equation system over $T$ is a finite set of equations over $T$. The set of equation systems over Term, denoted Eqs, is referred to as the set of concrete equation systems. Abstract equation systems involve equations over MTerm.

Definition 2.1 (Abstract equation system) An abstract equation system $\mathrm{AEqs}_{V}$, for a set of variables $V$, is a triple $\left\langle\mathcal{E} ; a n n_{\mathrm{AEqs}} ; \Delta\right\rangle$ such that $\mathcal{E}$ is an equation system over the set of mixed terms MTerm, with $\operatorname{Var}(\mathcal{E}) \subseteq V, \Delta \subseteq \wp(\operatorname{AVar}(\mathcal{E}))$ a sharing component, and $a n n_{\mathrm{AEqs}}$ an annotation mapping, ann $n_{\mathrm{AEqs}}: \mathrm{A} \operatorname{Var}(\mathcal{E}) \rightarrow \mathcal{A}$, where $\mathcal{A}$ is a lattice of annotations.

The set $V$ ( $\subseteq$ Var) of program variables that are of interest may be omitted as a subscript in $\mathrm{AEqs}_{V}$ when it is clear from the context. In the examples below, we use an implicit representation of the associated annotation mapping $a n n_{\mathrm{AEqs}}$ by annotating the occurrences of each abstract variable @ in AEqs to indicate the value of $a n n_{\mathrm{AEqs}}(@)$ : e.g., @f ${ }^{f} @^{l}$, and $@^{a}$ denote respectively that $a n n_{\mathrm{AEqs}}(@)$ is $f, l$, and $a$. The sharing component uses the set-sharing representation of Jacobs and Langen $[23,24]$. Each set $\left\{@_{i_{1}}, \ldots, @_{i_{n}}\right\}$ indicates that the terms represented by the abstract variables $@_{i_{1}}, \ldots, @_{i_{n}}$ can share one or more variables. For ease of implementation, abstract equation systems are always reduced to their so-called solution solved form.

Definition 2.2 (Solution Solved Form) An abstract equation system $\mathrm{AEqs}_{V}$ is in solution solved form if it contains for each $X \in V$ one equation of the form $X=t$ such that $\operatorname{Var}(t)=\emptyset$.

Example 2.1 For $V=\{A, B, C, D, E\}$,

$$
\mathrm{AEqs}_{V}=\left\langle\left\{\begin{array}{l}
A=@_{1}^{f}, B=f\left(@_{7}^{l}, g\left(@_{1}^{f}\right), @_{1}^{f}, @_{3}^{a}\right), \\
C=@_{5}^{f}, D=@_{4}^{l}, E=f\left(@_{4}^{l}\right)
\end{array}\right\} ;\left\{\begin{array}{l}
\left\{@_{1}^{f}\right\},\left\{@_{3}^{a}\right\}, \\
\left\{@_{1}^{f}, @_{4}^{l}, @_{5}^{f}\right\},\left\{@_{5}^{f}\right\}
\end{array}\right\},,\right.
$$


is an abstract equation system in solution solved form. It represents many concrete equation systems. For example

$$
\mathrm{Eqs}_{V}=\left\{\begin{array}{l}
A=X, B=f(a, g(X), X, n(Y, Y)) \\
C=X, D=h(X), E=f(h(X))
\end{array}\right\},
$$

is represented via the abstract term replacement $[4,6,10]$

$$
\mu: @_{1}^{f} \mapsto X, @_{3}^{a} \mapsto n(Y, Y), @_{4}^{l} \mapsto h(X), @_{5}^{f} \mapsto X, @_{7}^{l} \mapsto a .
$$

Specifications of operations for such abstract equation systems are in [6]. The order relation is based on a generalization of the well-known 'less general than' relation for the equations component, the 'subset-ordering' relation for the sharing component, and the 'order relation of the lattice of annotations' for the pointwise ordering of the annotation mappings. The upper-bound operation is based on a generalization of the anti-unification algorithm [29] for the equations component, the union operation for the sharing component, and the lub operation of the lattice of annotations for the annotation mappings.

The depth of an AEqs is defined in terms of the usual definition of term depth, denoted $t$-depth. The t-depth of an abstract or concrete variable is 0 ; the $t$-depth of a constant is 1 ; the t-depth of a compound term is $1+$ the maximum of the $t$-depths of its subterms. If AEqs is an abstract equation system in solution solved form and $n$ the maximum $t$ depth of the abstract terms occurring in the right-hand sides of the equations component, then the depth of AEqs is defined to be $n$, if every abstract variable occurring at depth $n$ has only one occurrence in the equation component, otherwise the depth is $n+1$. From this definition it follows that an AEqs of depth $\mathrm{n}$ may have 'same-value' or 'structural' information at depths $<n$ only (e.g. $\left\{A=@_{1}, B=@_{1}\right\}$ and $\{C=n i l\}$ have depth 1 , but $\left\{A=@_{1}, B=@_{2}, C=@_{3}\right\}$ has depth 0$)^{1}$. When imposing the depth bound on an abstract equation system, new abstract variables are introduced and the sharing and annotation component need to be updated. This is an easy operation which is based on techniques also used in the definition of the least upper-bound [6].

The set of abstract equation systems is denoted $\mathcal{A} E q s(k, \mathcal{A})$. The first parameter, $k$, is the depth-bound imposed on abstract terms and the second, $\mathcal{A}$, is the lattice of annotations used (e.g., $\mathcal{A}=\{f, l, a\}$, in which case the abstract variables of $\mathrm{AEqs}_{V}$ that do not occur in $\Delta$ (i.e., definitely ground abstract variables) must have annotation $l$; if $\mathcal{A}=\{f, a\}$ or $\mathcal{A}=\{a\}$, then ground abstract variables have annotation $a$; if $\mathcal{A}=\{a\}$, the annotation mapping is in fact redundant). A bottom abstract equation system, $\perp$, is also added to $\mathcal{A} E q s(k, \mathcal{A})$. Alternative representations for the sharing information are possible, e.g. the pair-sharing representation of the domain of Søndergaard [38] where $\Delta$ is a binary symmetric relation on $\operatorname{AVar}(\mathcal{E})$; the set $\mathcal{A}$ should in that case be extended with an explicit annotation $g$ for definitely ground terms, the $l$ annotation on the other hand can be left out since the sharing component provides linearity information (absence of reflexive pairs). Thus, the underlying sharing analysis could be considered as another parameter of the domain $\mathcal{A} E q s(k, \mathcal{A})$.

For $S S \subseteq$ AVar, we define $\Delta(S S)=\{S \in \Delta \mid S S \cap S \neq \emptyset\}$. In the notation of Jacobs and Langen [24], $\Delta(S S)$ corresponds to $\operatorname{rel}(S S, \Delta)$, the set of sharing groups relevant w.r.t.

\footnotetext{
${ }^{1}$ The motivation for the depth definition, which may seem a bit arbitrarily chosen, is that we want depth 0 to correspond with the basic mode and sharing domains, which do not express any structural or term-equality information.
} 
$S S$. For $S S \subseteq$ AVar, we define $\triangle \mid S S=\{S \cap S S \mid S \in \Delta \& S \cap S S \neq \emptyset\}$, the projection on $S S$ of $\Delta$. If we want to indicate explicitly that a term $\tau$ contains only the variables $\left\{@_{1}, \ldots, @_{n}\right\}$, with $(n \geq 0)$, and no other variables, $\tau$ is denoted by $\tau\left[@_{1}, \ldots, @_{n}\right]$. Let AEqs $=\left\langle\mathcal{E} ; a n n_{\mathrm{AEqs}} ; \Delta\right\rangle$ be an abstract equation system. We define the following predicates on the abstract variables occurring in AEqs:

free $_{\mathrm{AEqs}}(@) \Leftrightarrow a n n_{\mathrm{AEqs}}(@)=f$

$\operatorname{linear}_{\mathrm{AEqs}}(@) \Leftrightarrow a n n_{\mathrm{AEqs}}(@) \leq l$;

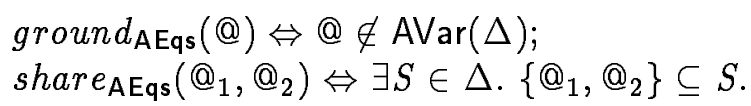

\subsection{The Unification Algorithm}

The basic strategy, also applied in $[10,11]$, is to systematically mimic each step in (an extended) Martelli-Montanari unification algorithm [33]. Equations on mixed terms are rewritten by the algorithm to a set of equations which are either in solution solved form or involve abstract terms only; the latter equations are then solved in the set-sharing domain augmented with the annotation mapping. By presenting the abstract unification algorithm as a high-level equation solving algorithm, its correctness proof can be split into verifying the rewriting system and proving the correctness of the underlying domain for sharing and mode analysis [6].

Algorithm 1 (Abstract unification) Let AEqs $=\left\langle\mathcal{E} ; a n n_{\mathrm{AEqs}} ; \Delta\right\rangle$ be an abstract equation system (in solution solved form) and $\zeta$ a finite sequence of equations on mixed terms over $\operatorname{Var}(\mathcal{E}) \cup \operatorname{A} \operatorname{Var}(\mathcal{E})$.

1. Replace all concrete variables in $\zeta$ by their corresponding abstract term description in AEqs (i.e., compute $\zeta^{\prime}=\zeta$ o $\mathrm{mgu}(\mathcal{E})$ ).

2. Apply the Abstract rewriting Algorithm 2 to $\left\langle\zeta^{\prime} ; \mathrm{AEqs}\right\rangle$.

3. If the result of the previous step is $\left\langle\zeta^{\prime \prime} ; \mathrm{AEqs}^{\prime \prime}\right\rangle(\neq \perp)$, then solve each of the abstract equations in $\zeta^{\prime \prime}$ successively using Algorithm 3.

4. Impose depth-bound $k$ on the resulting abstract equation system.

Note that infinite descending chains exist in the domain. For termination of the overall abstract interpretation algorithm [3], it would be sufficient to impose the depth-bound only on procedure-entry to (recursive) clauses. However, in order to evaluate the precision obtained for different choices of $k$ in a consistent way, the system should impose the depth-bound at each program point. We experimented with both variants of the system.

The abstract rewriting algorithm differs from the Martelli-Montanari algorithm in that it has a pair, $\langle\zeta ;$ AEqs $\rangle$, consisting of a sequence of equations and an abstract equation system as input/output parameter, and not only a sequence of equations, because rewriting the sequence of equations $\zeta$ can affect AEqs through the application of substitutions.

Algorithm 2 (Abstract rewriting) Let AEqs $=\left\langle\mathcal{E} ; a n n_{\mathrm{AEqs}} ; \Delta\right\rangle$ be an abstract equation system and $\zeta$ a finite sequence of equations on terms over $\mathrm{AVar}(\mathcal{E})$. Non-deterministically choose an equation from $\zeta$. The action taken by the algorithm is determined by the form of the equation. The algorithm terminates when there is no equation on which a step applies. If the rewriting fails, $\perp$ is returned.

1. $\langle @=@:: \zeta ; \mathrm{AEqs}\rangle \stackrel{\text { remove }}{\rightarrow}\langle\zeta ; \mathrm{AEqs}\rangle$.

2. $\langle @=t:: \zeta ; \mathrm{AEqs}\rangle \stackrel{\text { fail }}{\rightarrow} \quad \perp \quad$ if $@ \in \operatorname{AVar}(t) \& t \neq 0$. 


$$
\begin{aligned}
& \text { 3. }\langle @=t:: \zeta ; \mathrm{AEqs}\rangle \quad \stackrel{\text { subst }}{\rightarrow} \quad\left\langle @=t:: \zeta[@ \leftarrow t] ;\left\langle\mathcal{E}[@ \leftarrow t] ; a n n_{\mathrm{AEqs}} ; \Delta\right\rangle\right\rangle \\
& \text { if } @ \notin \operatorname{AVar}(t) \& \\
& @ \in A \operatorname{Var}(\zeta) \cup \operatorname{AVar}(\mathcal{E}) \text {. } \\
& \text { 4. }\langle t=@:: \zeta ; \mathrm{AEqs}\rangle \stackrel{\text { switch }}{\longrightarrow}\langle @=t:: \zeta ; \mathrm{AEqs}\rangle \quad \text { if } t \notin \mathrm{AVar} . \\
& \text { 5. }\left\langle f\left(s_{1}, \ldots, s_{n}\right)=g\left(t_{1}, \ldots, t_{m}\right) \quad:: \zeta ; \text { AEqs }\right\rangle \\
& \stackrel{\text { peel }}{\longrightarrow} \quad\left\langle\left\{s_{1}=t_{1}, \ldots, s_{n}=t_{n}\right\} \cup \zeta ; \text { AEqs }\right\rangle \quad \begin{array}{l}
\text { if } f / n \doteq g / m, \\
\stackrel{\text { fail }}{\longrightarrow}
\end{array}
\end{aligned}
$$

The resulting sequence of equations, $\zeta^{\prime \prime}$, consists of equations on terms over AVar, the left hand side of which is an abstract variable that does not occur in the resulting abstract equation system AEqs" nor in the right hand sides of the equations of $\zeta^{\prime \prime}$ (we say that $\zeta^{\prime \prime}$ is in abstract solved form). There are two kinds of equations that need to be solved in the underlying sharing and mode domain : $@_{0}=@_{1}$ and $@_{0}=\tau\left[@_{1}, \ldots, @_{n}\right](n \geq 0)$. For ease of presentation, the next algorithm only handles the basic case $@_{0}=@_{1}$; the generalization for $@_{0}=\tau\left[@_{1}, \ldots, @_{n}\right]$ is in $[6]$.

Algorithm 3 (General solve) Let $\mathrm{AEqs}=\left\langle\mathcal{E} ; a n n_{\mathrm{AEqs}} ; \Delta\right\rangle$ be an abstract equation system and $@_{0}=@_{1}:: \zeta$ a finite sequence of equations on terms over AVar. Solving the abstract equation $@_{0}=@_{1}$ yields the abstract equation system $\mathrm{AEqs}{ }^{\prime}=\left\langle\mathcal{E} ;\right.$ ann $\left.n_{\mathrm{AEqs}} ; \Delta^{\prime}\right\rangle$.

For the annotation lattice $\mathcal{A}=\{a\}$, the basic set-sharing unification algorithm of [23, 24] is used, i.e.,

$$
\begin{aligned}
\Delta^{\prime}=\quad \text { if } & \text { ground }_{\mathrm{AEqs}}\left(@_{0}\right) \vee \operatorname{ground}_{\mathrm{AEqs}}\left(@_{1}\right) \quad(o) \\
\text { then } & \Delta \backslash \Delta\left(\left\{@_{0}, @_{1}\right\}\right) \\
\text { else } & \Delta \backslash \Delta\left(\left\{@_{0}, @_{1}\right\}\right) \cup \\
& \left\{\left(S_{0} \cup S_{1}\right) \begin{array}{l}
\text { for } i=0,1, S_{i}=\cup S S \\
\text { where } S S \subseteq \Delta\left(\left\{@_{i}\right\}\right) \& S S \neq \emptyset .
\end{array}\right\} \backslash \operatorname{AVar}(\mathcal{E} \cup \zeta)
\end{aligned}
$$

and the annotation mapping ann $n_{\mathrm{AEqs}^{\prime}}$ trivially maps all abstract variables to a.

For the annotation lattice $\mathcal{A}=\{f, l, a\}$, the line $S_{i}=\cup S S$ in the definition of $\Delta^{\prime}$ above is replaced by

$$
\begin{array}{ll}
\text { if } \quad & \text { free }_{\mathrm{AEqs}}\left(@_{0}\right) \vee \text { free }_{\mathrm{AEqs}}\left(@_{1}\right) \vee \\
& \left(\text { linear }_{\mathrm{AEqs}}\left(@_{1-i}\right) \& \neg \text { share } \mathrm{AEqs}_{\mathrm{AE}}\left(@_{0}, @_{1}\right)\right) \\
\text { then } & S_{i} \in \Delta\left(\left\{@_{i}\right\}\right) \\
\text { else } & S_{i}=\cup S S
\end{array}
$$

and the new annotation mapping is defined by the following case analysis:

(1) for @ $\in \operatorname{AVar}(\mathcal{E}) \backslash A \operatorname{Var}\left(\Delta^{\prime}\right)$ (ground variable) :

$$
a n n_{\mathrm{AEqs}}(@)=l, \quad \text { (annotation for ground variable) }
$$

(2) for $@_{1}$, if $@_{1} \in \operatorname{AVar}\left(\Delta^{\prime}\right)$ :

$$
\begin{aligned}
& a n n_{\mathrm{AEqs}}\left(@_{1}\right)=\text { if } \operatorname{free}_{\mathrm{AEqs}}\left(@_{1}\right) \& \text { free }_{\mathrm{AEqs}}\left(@_{0}\right) \\
& \text { then ann } n_{\mathrm{AEqs}}\left(@_{1}\right) \text { (freeness and linearity preserved) } \\
& \text { else if linear } \mathrm{AEqs}\left(@_{0}\right) \& \neg \text { share }_{\mathrm{AEqs}}\left(@_{0}, @_{1}\right) \\
& \text { then lub }\left(a n n_{\mathrm{AEqs}}\left(@_{1}\right), l\right) \text { (linearity preserved) } \\
& \text { else } a \text {, }
\end{aligned}
$$


(3) for @ $\in \operatorname{AVar}\left(\Delta^{\prime}\right)$ such that $@ \notin\left\{@_{0}, @_{1}\right\}$ :

$$
\begin{aligned}
& a n n_{\mathrm{AEqs}}(@)=\text { if }\left(\neg \operatorname{share}_{\mathrm{AEqs}}\left(@_{1}, @\right) \& \neg \operatorname{share}_{\mathrm{AEqs}}\left(@_{0}, @\right)\right) \\
& \vee\left(\text { free }_{\mathrm{AEqs}}\left(@_{1}\right) \& \operatorname{free}_{\mathrm{AEqs}}\left(@_{0}\right) \& \operatorname{free}_{\mathrm{AEqs}}(@)\right) \\
& \vee\left(\text { free }_{\mathrm{AEqs}}\left(@_{0}\right) \&-\text { share }_{\mathrm{AEqs}}\left(@_{0}, @\right)\right) \\
& \vee\left(\text { free }_{\mathrm{AEqs}}\left(@_{1}\right) \& \neg \text { share }_{\mathrm{AEqs}}\left(@_{1}, @\right)\right) \\
& \text { then ann } n_{\mathrm{AEqs}}(@) \text { (freeness and linearity preserved) } \\
& \text { else } \\
& \text { if }\left(\text { linear }_{\mathrm{AEqs}}\left(@_{0}\right) \& \neg \text { share }_{\mathrm{AEqs}}\left(@_{0}, @_{)}\right)\right. \\
& \vee\left(\text { linear }_{\mathrm{AEqs}}\left(@_{1}\right) \& \neg \text { share }_{\mathrm{AEqs}}\left(@_{1}, @\right)\right) \\
& \text { then } \operatorname{lub}\left(a n n_{\mathrm{AEqs}}(@), l\right) \text { (linearity preserved) } \\
& \text { else } a
\end{aligned}
$$

We do not need the annotation of $@_{0}$, since $@_{0} \notin A \operatorname{Var}(\mathcal{E} \cup \zeta)$.

For the annotation lattices $\mathcal{A}=\{l, a\}$ and $\mathcal{A}=\{f, a\}$, we use similar definitions as for $\mathcal{A}=\{f, l, a\} ;$ the optimization rules using freeness, resp. linearity, are not applied. For $\mathcal{A}=\{f, a\}$, the annotation for a ground variable is a.

As far as we know, the optimization based on freeness information at line $\left(^{*}\right)$ was first suggested in $[34,36]$ for the Sharing+Free domain, while the optimization based on linearity information at line $\left({ }^{* *}\right)$ was first suggested in [4].

Example 2.2 Consider annotation lattice $\{a, l, f\}$, depth-bound $k=2$, and $\mathcal{E}=\{X=$ $\left.f\left(@_{1}^{f}, @_{2}^{l}\right), Y=@_{3}^{f}, Z=@_{4}^{f}\right\}, \Delta=\left\{\left\{@_{1}^{f}\right\},\left\{@_{2}^{l}\right\},\left\{@_{3}^{f}\right\},\left\{@_{4}^{f}\right\}\right\}$, and $\zeta=\{X=f(Y, Z)\}$. After step 1 of Algorithm 1, we have the singleton set $\zeta^{\prime}=\left\{f\left(@_{1}^{f}, @_{2}^{l}\right)=f\left(@_{3}^{f}, @_{4}^{f}\right)\right\}$ of abstract equations to be solved. After step 2, we have $\mathcal{E}^{\prime \prime}=\left\{X=f\left(@_{3}^{f}, @_{4}^{f}\right), Y=\right.$ $\left.@_{3}^{f}, Z=@_{4}^{f}\right\}, \Delta^{\prime \prime}=\Delta$, and $\zeta^{\prime \prime}=\left\{@_{1}^{f}=@_{3}^{f}, @_{2}^{l}=@_{4}^{f}\right\}$. After step 3, we obtain $\mathcal{E}^{\prime \prime \prime}=\left\{X=f\left(@_{3}^{f}, @_{4}^{l}\right), Y=@_{3}^{f}, Z=@_{4}^{l}\right\}$ and $\Delta^{\prime \prime \prime}=\left\{\left\{@_{3}^{f}\right\},\left\{@_{4}^{l}\right\}\right\}$ as the resulting system, since step 4 is a null operation.

The justification of the case analysis defining the annotation mapping is based on the following lemma which specifies sufficient conditions for the freeness and linearity properties of a term $r$ to be preserved under application of the most general unifier of two concrete terms $t$ and $s$.

Lemma $2.1([5,6])$ Let $t$ and $s$ be unifiable terms, $\theta=\operatorname{mgu}(t, s)$ idempotent and relevant, and $r$ an arbitrary term ( $r$ may be a subterm of $t$ or $s$ ). It holds that

1. if $\operatorname{Var}(r) \cap(\operatorname{Var}(t) \cup \operatorname{Var}(s))=\emptyset$ then $r \theta=r$,

2. if free $(t)$ \& free $(s)$ then $f r e e(r) \rightarrow f r e e(r \theta)$,

3. if free $(t) \& \operatorname{Var}(t) \cap \operatorname{Var}(r)=\emptyset$ then $f r e e(r) \rightarrow$ free $(r \theta)$ \& linear $(r) \rightarrow \operatorname{linear}(r \theta)$,

4. if linear $(t) \& \operatorname{Var}(t) \cap \operatorname{Var}(r)=\emptyset$ then linear $(r) \rightarrow \operatorname{linear}(r \theta)$. 


\section{Experimental Evaluation}

The experimental tool (implemented in SICStus Prolog) is based on the PLAI system, which is a domain independent framework and environment for developing global analyzers based on abstract interpretation. The PLAI system has been used for a variety of applications, e.g., [8, 13, 17, 36]. It was originally developed for logic programs [22], was subsequently provided with a more efficient fix-point algorithm $[35,36,37$, and extended for handling CLP languages $[18,19]$. The latter feature is not used in the present evaluation.

\subsection{Results}

Table 1 lists the benchmark programs together with some of their characteristics, namely $\mathrm{Cl}$, the number of clauses, and $\mathrm{Pr}$, the number of predicates defined in the program. A brief description of each program is given in Appendix B. Several of the benchmarks are unnormalized programs - arguments of heads and calls are allowed to be non-variable terms - however browse, bid, read, boyer, and ann are semi-normalized - arguments of calls to predicates are variables. Analyzing semi-normalized programs is often faster since usually less call patterns have to be analyzed. The saving is normally larger than the overhead caused by the extra local variables introduced by semi-normalization. This is illustrated in Table 1 by comparing the browse_u (unnormalized) with the browse (seminormalized) benchmark.

For various parameter settings, Table 1 further shows the number of call patterns (Pat) that are analyzed (if equal to the number of predicates, then - in the absence of dead code - each predicate is analyzed for one call pattern), the time for the analysis and the memory needed by the analysis. The timings are measured on a SPARCstation IPC and include time for stack-shifts and garbage collection. (The tables in Appendix C show the analysis time and the time for stack-shifts and garbage collection separately.) The memory used by PLAI at start-up time is $3.14 \mathrm{Mb}$.

Table 2 summarizes - by making the summation over all benchmark programs experiments aimed at determining the importance of the different special cases in the abstract unification (Algorithm 3). The meaning of the columns is as follows :

FR: Percentage of applications of freeness rule $(*)$.

L1R: Percentage of applications of linear rule (one-side) $\left.{ }^{* *}\right)$.

L2R: Percentage of applications of linear rule (both-sides) $\left.{ }^{* *}\right)$.

GrR: Percentage of applications of groundness rule (o).

NoR: Percentage of applications of general rule.

Unif: Total number of calls for unification operations.

(The full tables corresponding to the summary table are in Appendix D.)

Measurements have been done for four annotation lattices $\{a, l, f\},\{a, l\},\{a, f\}$ and $\{a\}$. In the $\{a, l\}$ case for instance, the freeness rule is never activated, hence the 0.0 in the FR-column. For all four lattices, the use of the groundness rule varies from more than $90 \%$ for the programs grammar, bid, deriv, kalah, read, and ch_db_gr, down to about $20 \%$ for boyer and even less for mapcolor'. For the lattice $\{a, l, f\}$, the use of the freeness rule varies from less than $5 \%$ in bid and ch_db_gr to more than $40 \%$ in mapcolor', rdtok, boyer, and icomp. The use of linearity is rather marginal in the $\{a, l, f\}$ domain, but 


\begin{tabular}{|c|c|c|c|c|c|c|c|c|c|c|c|c|c|c|}
\hline \multirow[b]{2}{*}{ Program } & \multirow[b]{2}{*}{$\mathrm{Cl}$} & \multirow[b]{2}{*}{$\operatorname{Pr}$} & \multicolumn{3}{|c|}{ alf-0 } & \multicolumn{3}{|c|}{ alf-1 } & \multicolumn{3}{|c|}{ alf-2 } & \multicolumn{3}{|c|}{ alf-3 } \\
\hline & & & Pat & sec & $\mathrm{Mb}$ & Pat & sec & $\mathrm{Mb}$ & Pat & sec & $\mathrm{Mb}$ & Pat & sec & $\mathrm{Mb}$ \\
\hline serialize & 11 & 5 & 8 & 3.80 & 4.1 & 8 & 3.90 & 4.1 & 8 & 3.86 & 4.1 & 8 & 3.79 & 4.1 \\
\hline init_vars & 12 & 7 & 9 & 3.22 & 4.1 & 9 & 5.00 & 4.1 & 9 & 4.77 & 4.1 & 9 & 4.81 & 4.1 \\
\hline mapcolor' & 12 & 8 & 13 & 2.53 & 4.1 & 22 & 81.29 & 4.1 & 24 & 110.29 & 4.1 & 29 & 1539.20 & 4.2 \\
\hline mapcolor & 13 & 8 & 8 & 0.76 & 3.5 & 19 & 2.28 & 4.1 & 27 & 4.02 & 4.1 & 36 & 6.54 & 4.2 \\
\hline grammar & 14 & 6 & 6 & 0.46 & 3.3 & 6 & 0.61 & 3.5 & 6 & 0.57 & 3.5 & 6 & 0.57 & 3.5 \\
\hline browse & 32 & 15 & 21 & 12.21 & 4.3 & 24 & 28.10 & 4.3 & 37 & 11.43 & 4.3 & 52 & 17.26 & 4.4 \\
\hline browse_u & 32 & 15 & 27 & 29.10 & 4.3 & 30 & 45.55 & 4.3 & 42 & 11.78 & 4.3 & 57 & 17.37 & 4.4 \\
\hline bid & 53 & 22 & 24 & 2.69 & 4.2 & 29 & 3.88 & 4.2 & 34 & 6.02 & 4.2 & 39 & 7.83 & 4.3 \\
\hline rdtok & 55 & 18 & 39 & 22.11 & 4.7 & 39 & 23.61 & 4.7 & 40 & 23.68 & 4.7 & 41 & 23.81 & 4.7 \\
\hline deriv & 62 & 15 & 31 & 7.93 & 4.3 & 40 & 10.85 & 4.4 & 46 & 13.18 & 4.4 & 49 & 14.04 & 4.5 \\
\hline kalah & 78 & 41 & 53 & 10.77 & 4.4 & 54 & 18.96 & 4.5 & 61 & 29.59 & 4.5 & 90 & 59.77 & 4.7 \\
\hline $\operatorname{tarjan}$ & 89 & 36 & 70 & 29.86 & 4.8 & 96 & 61.01 & 5.1 & 126 & 124.53 & 5.6 & 314 & 1171.12 & 11.1 \\
\hline read & 90 & 24 & 27 & 20.76 & 4.6 & 43 & 62.50 & 5.2 & 58 & 101.56 & 5.7 & 80 & 190.96 & 6.9 \\
\hline ch_db_gr & 130 & 45 & 70 & 19.27 & 4.5 & 82 & 27.65 & 4.7 & 93 & 45.00 & 4.8 & 108 & 63.03 & 4.9 \\
\hline ch_db & 130 & 45 & 75 & 22.59 & 4.5 & 89 & 31.70 & 4.7 & 102 & 50.51 & 4.9 & 117 & 68.89 & 5.0 \\
\hline peephole & 134 & 16 & 42 & 35.70 & 4.6 & 42 & 46.18 & 4.6 & 36 & 22.95 & 4.5 & 36 & 21.83 & 4.5 \\
\hline boyer & 138 & 28 & 49 & 21.08 & 4.5 & 69 & 54.70 & 4.8 & 94 & 114.39 & 5.3 & 125 & 221.43 & 5.8 \\
\hline icomp & 144 & 62 & 165 & 135.15 & 5.7 & 204 & 513.23 & 6.0 & 239 & 3750.10 & 6.2 & & & \\
\hline ann & 181 & 50 & 78 & 307.35 & 4.9 & & & & & & & & & \\
\hline
\end{tabular}

\begin{tabular}{|c|c|c|c|c|c|c|c|c|c|c|c|c|c|c|}
\hline \multirow[b]{2}{*}{ Program } & \multirow[b]{2}{*}{$\mathrm{Cl}$} & \multirow[b]{2}{*}{$\operatorname{Pr}$} & \multicolumn{3}{|c|}{ alf-0 } & \multicolumn{3}{|c|}{ al-0 } & \multicolumn{3}{|c|}{ af-0 } & \multicolumn{3}{|c|}{$a-0$} \\
\hline & & & Pat & sec & $\mathrm{Mb}$ & Pat & sec & $\mathrm{Mb}$ & Pat & sec & $\mathrm{Mb}$ & Pat & sec & $\mathrm{Mb}$ \\
\hline serialize & 11 & 5 & 8 & 3.80 & 4.1 & 7 & 3.73 & 4.1 & 8 & 4.03 & 4.1 & 13 & 24.79 & 4.2 \\
\hline init_vars & 12 & 7 & 9 & 3.22 & 4.1 & 9 & 3.15 & 4.1 & 9 & 3.12 & 4.1 & 9 & 3.08 & 4.1 \\
\hline mapcolor' & 12 & 8 & 13 & 2.53 & 4.1 & 13 & 2.63 & 4.1 & 13 & 2.55 & 4.1 & 13 & 3.24 & 4.1 \\
\hline mapcolor & 13 & 8 & 8 & 0.76 & 3.5 & 8 & 0.85 & 3.5 & 8 & 0.80 & 3.5 & 8 & 1677.30 & 4.1 \\
\hline grammar & 14 & 6 & 6 & 0.46 & 3.3 & 6 & 0.46 & 3.3 & 6 & 0.44 & 3.3 & 6 & 0.42 & 3.3 \\
\hline browse & 32 & 15 & 21 & 12.21 & 4.3 & 21 & 12.11 & 4.2 & 24 & 13.62 & 4.3 & 28 & 640.16 & 4.3 \\
\hline browse_u & 32 & 15 & 27 & 29.10 & 4.3 & 27 & 29.15 & 4.2 & 30 & 30.11 & 4.3 & 33 & 256.18 & 4.3 \\
\hline bid & 53 & 22 & 24 & 2.69 & 4.2 & 24 & 2.74 & 4.2 & 24 & 2.69 & 4.2 & 24 & 2.73 & 4.2 \\
\hline rdtok & 55 & 18 & 39 & 22.11 & 4.7 & 32 & 16.11 & 4.6 & 39 & 18.91 & 4.6 & 39 & 19.78 & 4.6 \\
\hline deriv & 62 & 15 & 31 & 7.93 & 4.3 & 31 & 8.08 & 4.3 & 31 & 7.89 & 4.3 & 31 & 7.97 & 4.3 \\
\hline kalah & 78 & 41 & 53 & 10.77 & 4.4 & 53 & 10.73 & 4.4 & 53 & 10.72 & 4.4 & 53 & 10.52 & 4.4 \\
\hline tarjan & 89 & 36 & 70 & 29.86 & 4.8 & 69 & 30.69 & 4.8 & 70 & 29.52 & 4.8 & 73 & 43.43 & 4.8 \\
\hline read & 90 & 24 & 27 & 20.76 & 4.6 & 27 & 21.13 & 4.6 & 27 & 20.13 & 4.6 & 29 & 20.53 & 4.6 \\
\hline ch_db_gr & 130 & 45 & 70 & 19.27 & 4.5 & 70 & 19.45 & 4.5 & 70 & 18.60 & 4.5 & 70 & 18.69 & 4.5 \\
\hline ch_db & 130 & 45 & 75 & 22.59 & 4.5 & 74 & 22.82 & 4.5 & 75 & 88.88 & 4.6 & 76 & 478.87 & 4.6 \\
\hline peephole & 134 & 16 & 42 & 35.70 & 4.6 & 44 & 37.74 & 4.6 & 39 & 27.33 & 4.5 & 45 & 101.76 & 4.6 \\
\hline boyer & 138 & 28 & 49 & 21.08 & 4.5 & 46 & 21.64 & 4.5 & 49 & 112.13 & 4.5 & 46 & 113.36 & 4.5 \\
\hline icomp & 144 & 62 & 165 & 135.15 & 5.7 & 154 & 136.55 & 5.6 & 165 & 132.69 & 5.6 & 149 & 183.17 & 5.5 \\
\hline ann & 181 & 50 & 78 & 307.35 & 4.9 & 78 & 310.73 & 4.9 & 76 & 306.59 & 4.9 & 92 & 477.82 & 5.0 \\
\hline
\end{tabular}

Table 1: Timings and memory usage 


\begin{tabular}{|l||r|r|r|r|r|r|}
\hline Ann-k & FR & L1R & L2R & GrR & NoR & Unif \\
\hline \hline alf-0 & 29.4 & 2.3 & 3.7 & 59.7 & 4.9 & 24855 \\
alf-1 & 27.3 & 1.9 & 4.8 & 61.7 & 4.2 & 34490 \\
alf-2 & 24.7 & 1.7 & 6.3 & 63.9 & 3.4 & 45664 \\
\hline al-0 & 0.0 & 14.7 & 19.9 & 60.3 & 5.0 & 23866 \\
al-1 & 0.0 & 12.4 & 21.0 & 62.3 & 4.3 & 33334 \\
al-2 & 0.0 & 10.0 & 21.9 & 64.6 & 3.4 & 44114 \\
\hline af-0 & 27.3 & 0.0 & 0.0 & 63.3 & 9.4 & 22607 \\
af-1 & 24.5 & 0.0 & 0.0 & 67.2 & 8.3 & 29770 \\
af-2 & 21.2 & 0.0 & 0.0 & 71.6 & 7.2 & 37971 \\
\hline a-0 & 0.0 & 0.0 & 0.0 & 63.8 & 36.2 & 22262 \\
a-1 & 0.0 & 0.0 & 0.0 & 67.8 & 32.2 & 29252 \\
a-2 & 0.0 & 0.0 & 0.0 & 71.9 & 28.1 & 37508 \\
\hline
\end{tabular}

Table 2: Application of optimization rules

is important in the $\{a, l\}$ domain; in both domains, the rule for both sides linear is the more frequently applied. In the $\{a, l, f\}$ domain, the general rule covers only about $5 \%$ of the cases - even less for larger depths - with percentages ranging from $0.0 \%$ for the serialize, init_vars, mapcolor, grammar, bid, deriv, kalah, and read programs to $13.6 \%$ for the icomp and $20.8 \%$ for the mapcolor' program. In the $\{a\}$ domain, the general rule is much more important with percentages ranging from less than $5 \%$ for bid and ch_db_gr to about $85 \%$ for mapcolor'.

Table 3 shows a few of our results concerning precision. For the annotation lattice $\{a, l, f\}$, it compares depth $0,1,2$, and 3 analyses with a depth $0^{*}$ analysis. The latter analysis only imposes the depth restriction upon procedure-entry. At other program points, abstract terms of arbitrary depth are derivable. The number of call patterns analyzed in the 0 and $0^{*}$ analyses is identical (see table at the end of Appendix $\mathrm{C}$ ); the analysis times are comparable except for the programs init_vars, mapcolor, and peephole for which the analysis $0^{*}$ is a factor 2 slower, the programs icomp and mapcolor' where $0^{*}$ is factors 3 and 6 slower respectively, and for the programs browse_u and ann where the analysis time for $0^{*}$ becomes unacceptable. To make a comparison w.r.t. precision possible, a single upper-bound has been computed from the results of the analysis of different call patterns. In the case that the analyses of different call patterns have incomparable results, the upper-bound computes a safe approximation which contains less precise information than the results of the individual analyses. Moreover, all results have been projected on the program variables. The meaning of the columns of Table 3 is as follows:

Gr: Number of program variables with mode ground.

Fr: Number of program variables with mode free.

Lin: Number of program variables with mode linear (but not ground).

Any: Number of program variables with mode any.

Sin: Number of singleton abstract sharing groups.

NSin: Number of non-singleton abstract sharing groups.

Str: Amount of structural information (number of function symbols).

Sval: Amount of term-equality information (multiple occurrences of abstract variables). 


\begin{tabular}{|c|c|c|c|c|c|c|c|c|c|c|c|}
\hline Program & Ann-k & $\mathrm{Gr}$ & $\mathrm{Fr}$ & Lin & Any & Sin & NSin & Str & Sval & $\mathrm{AvS}$ & $\mathrm{MxS}$ \\
\hline \multirow[t]{5}{*}{ serialize } & alf- $0 \downarrow 0$ & 40 & 40 & 82 & 13 & 98 & 25 & 0 & 0 & 4.39 & 7 \\
\hline & alf- $1 \downarrow 0$ & 40 & 40 & 82 & 13 & 98 & 25 & 0 & 3 & 4.39 & 7 \\
\hline & alf- $2 \downarrow 0$ & 40 & 40 & 82 & 13 & 98 & 25 & 0 & 3 & 4.39 & 7 \\
\hline & alf- $3 \downarrow 0$ & 40 & 40 & 82 & 13 & 98 & 25 & 0 & 3 & 4.39 & 7 \\
\hline & alf- $0^{*} \downarrow 0$ & 40 & 40 & 82 & 13 & 98 & 25 & 0 & 3 & 4.39 & 7 \\
\hline \multirow[t]{5}{*}{ init_vars } & alf- $0 \downarrow 0$ & 137 & 72 & 5 & 0 & 67 & 5 & 0 & 0 & 2.88 & 7 \\
\hline & alf- $1 \downarrow 0$ & 137 & 72 & 5 & 0 & 67 & 5 & 6 & 4 & 2.88 & 7 \\
\hline & alf- $2 \downarrow 0$ & 137 & 72 & 5 & 0 & 67 & 5 & 6 & 16 & 2.88 & 7 \\
\hline & alf- $3 \downarrow 0$ & 137 & 72 & 5 & 0 & 67 & 5 & 6 & 16 & 2.88 & 7 \\
\hline & alf $-0^{*} \downarrow 0$ & 137 & 72 & 5 & 0 & 67 & 5 & 6 & 16 & 2.88 & 7 \\
\hline \multirow[t]{5}{*}{ mapcolor' } & alf- $0 \downarrow 0$ & 11 & 13 & 1 & 58 & 42 & 101 & 0 & 0 & 6.81 & 25 \\
\hline & alf- $1 \downarrow 0$ & 11 & 13 & 1 & 58 & 42 & 101 & 20 & 0 & 13.57 & 49 \\
\hline & alf $-2 \downarrow 0$ & 11 & 13 & 1 & 58 & 42 & 101 & 33 & 0 & 15.14 & 49 \\
\hline & alf- $3 \downarrow 0$ & 11 & 13 & 1 & 58 & 42 & 94 & 49 & 3 & 17.10 & 96 \\
\hline & alf- $0 * \downarrow 0$ & 11 & 13 & 1 & 58 & 42 & 101 & 185 & 66 & 15.81 & 64 \\
\hline \multirow[t]{5}{*}{ mapcolor } & alf- $0 \downarrow 0$ & 52 & 25 & 6 & 10 & 33 & 10 & 0 & 0 & 3.31 & 10 \\
\hline & alf- $1 \downarrow 0$ & 52 & 25 & 6 & 10 & 33 & 10 & 20 & 0 & 3.46 & 10 \\
\hline & alf- $2 \downarrow 0$ & 52 & 25 & 6 & 10 & 33 & 10 & 42 & 0 & 3.77 & 10 \\
\hline & alf- $3 \downarrow 0$ & 52 & 25 & 6 & 10 & 33 & 10 & 66 & 2 & 4.00 & 10 \\
\hline & alf- $0 * \downarrow 0$ & 52 & 25 & 6 & 10 & 33 & 10 & 107 & 14 & 4.46 & 16 \\
\hline \multirow[t]{5}{*}{ grammar } & alf- $0 \downarrow 0$ & 32 & 13 & 7 & 0 & 20 & 0 & 0 & 0 & 1.54 & 5 \\
\hline & alf- $1 \downarrow 0$ & 37 & 13 & 2 & 0 & 15 & 0 & 13 & 0 & 1.88 & 5 \\
\hline & alf- $2 \downarrow 0$ & 37 & 13 & 2 & 0 & 15 & 0 & 17 & 4 & 1.88 & 5 \\
\hline & alf- $3 \downarrow 0$ & 37 & 13 & $\mathscr{2}$ & 0 & 15 & 0 & 18 & 9 & 1.88 & 5 \\
\hline & alf- $0 * \downarrow 0$ & 33 & 13 & 6 & 0 & 19 & 0 & 24 & 14 & 1.58 & 5 \\
\hline \multirow[t]{5}{*}{ browse } & alf- $0 \downarrow 0$ & 214 & 172 & 103 & 79 & 279 & 570 & 0 & 0 & 9.23 & 512 \\
\hline & alf- $1 \downarrow 0$ & 214 & 172 & 103 & 79 & 279 & 570 & 53 & 3 & 9.60 & 512 \\
\hline & alf $-2 \downarrow 0$ & 214 & 172 & 109 & 73 & 279 & 72 & 124 & 12 & 4.29 & 14 \\
\hline & alf- $3 \downarrow 0$ & 214 & 172 & 109 & 73 & 279 & 72 & 235 & 17 & 4.51 & 14 \\
\hline & alf- $0^{*} \downarrow 0$ & 214 & 172 & 103 & 79 & 279 & 570 & 338 & 55 & 9.38 & 512 \\
\hline \multirow[t]{5}{*}{ browse_u } & alf- $0 \downarrow 0$ & 176 & 125 & 97 & 76 & 245 & 551 & 0 & 0 & 9.26 & 512 \\
\hline & alf- $1 \downarrow 0$ & 176 & 125 & 97 & 76 & 245 & 551 & 43 & 3 & 9.63 & 512 \\
\hline & alf- $2 \downarrow 0$ & 176 & 125 & 103 & 70 & 245 & 53 & 99 & 7 & 3.94 & 14 \\
\hline & alf- $3 \downarrow 0$ & 176 & 125 & 103 & 70 & 245 & 53 & 192 & 7 & 4.15 & 14 \\
\hline & alf- $0^{*} \downarrow 0$ & 176 & 125 & 97 & 76 & 245 & 551 & 206 & 7 & 9.42 & 512 \\
\hline \multirow[t]{5}{*}{ bid } & alf- $0 \downarrow 0$ & 340 & 123 & 1 & 0 & 124 & 0 & 0 & 0 & 2.25 & 8 \\
\hline & alf- $1 \downarrow 0$ & 340 & 123 & 1 & 0 & 124 & 0 & 33 & 0 & 2.25 & 8 \\
\hline & alf- $2 \downarrow 0$ & 340 & 123 & 1 & 0 & 124 & 0 & 91 & 0 & 2.25 & 8 \\
\hline & alf- $3 \downarrow 0$ & 340 & 123 & 1 & 0 & 124 & 0 & 149 & 1 & 2.25 & 8 \\
\hline & alf $-0^{*} \downarrow 0$ & 340 & 123 & 1 & 0 & 124 & 0 & 284 & 0 & 2.25 & 8 \\
\hline \multirow[t]{5}{*}{ rdtok } & alf- $0 \downarrow 0$ & 337 & 320 & 145 & 43 & 498 & 46 & 0 & 0 & 2.86 & 7 \\
\hline & alf- $1 \downarrow 0$ & 337 & 320 & 147 & 41 & 496 & $4^{2}$ & 8 & 5 & 2.85 & 6 \\
\hline & alf- $2 \downarrow 0$ & 337 & 320 & 147 & 41 & 496 & $4^{2}$ & 8 & 5 & 2.85 & 6 \\
\hline & alf- $3 \downarrow 0$ & 337 & 320 & 147 & 41 & 496 & $4^{2}$ & 8 & 5 & 2.85 & 6 \\
\hline & alf- $0^{*} \downarrow 0$ & 337 & 320 & 145 & 43 & 498 & 46 & 14 & 3 & 2.88 & 7 \\
\hline \multirow[t]{5}{*}{ deriv } & alf- $0 \downarrow 0$ & 523 & 193 & 0 & 0 & 193 & 0 & 0 & 0 & 1.74 & 4 \\
\hline & alf- $1 \downarrow 0$ & 523 & 193 & 0 & 0 & 193 & 0 & 23 & 0 & 1.74 & 4 \\
\hline & alf- $2 \downarrow 0$ & 523 & 193 & 0 & 0 & 193 & 0 & 26 & 7 & 1.74 & 4 \\
\hline & alf- $3 \downarrow 0$ & 523 & 193 & 0 & 0 & 193 & 0 & 26 & 7 & 1.74 & 4 \\
\hline & alf $-0^{*} \downarrow 0$ & 523 & 193 & 0 & 0 & 193 & 0 & 8 & 5 & 1.74 & 4 \\
\hline \multirow[t]{5}{*}{ kalah } & alf- $0 \downarrow 0$ & 969 & 238 & 28 & 5 & 271 & 1 & 0 & 0 & 1.97 & 7 \\
\hline & alf- $1 \downarrow 0$ & 969 & 238 & 28 & 5 & 271 & 1 & 161 & 0 & 2.04 & 7 \\
\hline & alf- $2 \downarrow 0$ & 958 & 237 & 28 & 5 & 270 & 1 & 511 & 12 & 2.04 & 7 \\
\hline & alf- $3 \downarrow 0$ & 958 & 237 & 28 & 5 & 270 & 1 & 882 & 22 & 2.04 & 7 \\
\hline & alf- $0 * \downarrow 0$ & 969 & 238 & 28 & 5 & 271 & 1 & 358 & 17 & 1.97 & 7 \\
\hline
\end{tabular}

Table 3: Impact of structural information 


\begin{tabular}{|c|c|c|c|c|c|c|c|c|c|c|c|}
\hline Program & Ann-k & $\mathrm{Gr}$ & $\mathrm{Fr}$ & Lin & Any & Sin & NSin & Str & Sval & $\mathrm{AvS}$ & $\mathrm{MxS}$ \\
\hline \multirow[t]{5}{*}{ tarjan } & $\overline{\text { alf- } 0 \downarrow 0}$ & $\overline{1037}$ & $\overline{449}$ & 120 & $\overline{301}$ & $\overline{817}$ & $\overline{346}$ & 0 & 0 & $\overline{4.31}$ & $\overline{\overline{35}}$ \\
\hline & alf- $1 \downarrow 0$ & 1037 & 449 & 122 & 299 & 817 & 343 & 247 & 3 & 4.62 & 35 \\
\hline & alf- $2 \downarrow 0$ & 1037 & 449 & 122 & 299 & 817 & 343 & 489 & 211 & 4.62 & 35 \\
\hline & alf-3\0 & 1037 & 449 & 122 & 299 & 817 & 343 & 642 & 461 & 4.62 & 35 \\
\hline & alf- $0^{*} \downarrow 0$ & 1037 & 449 & 120 & 301 & 817 & 346 & 385 & 632 & 4.63 & 35 \\
\hline \multirow[t]{5}{*}{ read } & alf-0 00 & 1370 & 709 & 4 & 4 & 715 & 2 & 0 & 0 & 3.32 & 8 \\
\hline & alf- $1 \downarrow 0$ & 1370 & 709 & 4 & 4 & 715 & 2 & 133 & 1 & 3.32 & 8 \\
\hline & alf- $2 \downarrow 0$ & 1370 & 709 & 4 & 4 & 715 & 2 & 164 & 57 & 3.32 & 8 \\
\hline & alf- $3 \downarrow 0$ & 1370 & 709 & 4 & 4 & 715 & 2 & 172 & 103 & 3.32 & 8 \\
\hline & alf- $0^{*} \downarrow 0$ & 1370 & 709 & 4 & 4 & 715 & 2 & 325 & 102 & 3.32 & 8 \\
\hline \multirow[t]{5}{*}{ ch_db_gr } & alf-0 0 & 1106 & 212 & 29 & 20 & 259 & 16 & 0 & 0 & 2.31 & 8 \\
\hline & alf- $1 \downarrow 0$ & 1134 & 216 & 1 & 16 & 231 & 8 & 112 & 9 & 2.49 & 8 \\
\hline & alf- $2 \downarrow 0$ & 1134 & 216 & 1 & 16 & 231 & 8 & 210 & 18 & 2.49 & 8 \\
\hline & alf-3\0 & 1134 & 216 & 1 & 16 & 231 & 8 & 214 & 61 & 2.49 & 8 \\
\hline & alf- $0^{*} \downarrow 0$ & 1106 & 216 & 29 & 16 & 259 & 8 & 77 & 36 & 2.24 & 8 \\
\hline \multirow[t]{5}{*}{ ch_db } & alf-0 0 & 910 & 217 & 214 & 35 & 438 & 42 & 0 & 0 & 2.67 & 11 \\
\hline & alf- $1 \downarrow 0$ & 910 & 221 & 214 & 31 & 433 & 34 & 113 & 9 & 2.86 & 11 \\
\hline & alf- $2 \downarrow 0$ & 910 & 221 & 214 & 31 & 433 & 34 & 211 & 18 & 2.88 & 11 \\
\hline & alf- $3 \downarrow 0$ & 910 & 221 & 214 & 31 & 433 & 34 & 215 & 61 & 2.88 & 11 \\
\hline & alf $-0 * \downarrow 0$ & 910 & 221 & 214 & 31 & 438 & 34 & 77 & 36 & 3.12 & 19 \\
\hline \multirow[t]{5}{*}{ peephole } & alf- $0 \downarrow 0$ & 593 & 205 & 120 & 186 & 366 & 458 & 0 & 0 & 3.94 & 33 \\
\hline & alf- $1 \downarrow 0$ & 593 & 205 & 120 & 186 & 366 & 456 & 71 & 0 & 4.35 & 33 \\
\hline & alf $-2 \downarrow 0$ & 593 & 211 & 281 & 15 & 358 & 101 & 96 & 32 & 2.30 & 9 \\
\hline & alf- $3 \downarrow 0$ & 593 & 211 & 281 & 15 & 358 & 101 & 108 & 68 & 2.30 & 9 \\
\hline & alf $-0 * \downarrow 0$ & 593 & 205 & 120 & 186 & 366 & 458 & 94 & 89 & 4.40 & 33 \\
\hline \multirow[t]{5}{*}{ boyer } & alf-0 00 & 137 & 66 & 447 & 48 & 466 & 120 & 0 & 0 & 2.82 & 18 \\
\hline & alf- $1 \downarrow 0$ & 137 & 66 & 447 & 48 & 466 & 120 & 38 & 12 & 2.85 & 18 \\
\hline & alf $-2 \downarrow 0$ & 137 & 66 & 447 & 48 & 466 & 120 & 74 & 28 & 2.85 & 18 \\
\hline & alf- $3 \downarrow 0$ & 137 & 66 & 447 & 48 & 466 & 120 & 132 & 28 & 2.85 & 18 \\
\hline & alf- $0^{*} \downarrow 0$ & 137 & 66 & 447 & 48 & 466 & 120 & 579 & 35 & 2.85 & 18 \\
\hline \multirow[t]{4}{*}{ icomp } & alf- $0 \downarrow 0$ & 816 & 1032 & 131 & 1419 & 1927 & 2504 & 0 & 0 & 7.87 & 57 \\
\hline & alf- $1 \downarrow 0$ & 816 & 1032 & 131 & 1419 & 1927 & 2504 & 114 & 43 & 8.69 & 208 \\
\hline & alf- $2 \downarrow 0$ & 816 & 1032 & 131 & 1419 & 1927 & 2504 & 155 & 181 & 8.81 & 208 \\
\hline & alf $-0^{*} \downarrow 0$ & 816 & 1032 & 131 & 1419 & 1927 & 2504 & 248 & 230 & 8.69 & 208 \\
\hline
\end{tabular}

Table 3: Impact of structural information (continued)

\begin{tabular}{|c|c|c|c|c|c|c|c|c|c|c|c|}
\hline Program & Ann-k & $\mathrm{Gr}$ & Fr & Lin & Any & Sin & NSin & Str & Sval & $\mathrm{AvS}$ & $\mathrm{MxS}$ \\
\hline \multirow[t]{4}{*}{ serialize } & alf- $0 \downarrow 0$ & 40 & 40 & 82 & 13 & 98 & 25 & 0 & 0 & 4.39 & 7 \\
\hline & al- $0 \downarrow 0$ & 40 & 0 & 122 & 13 & 98 & 25 & 0 & 0 & 4.39 & 7 \\
\hline & af $-0 \downarrow 0$ & 40 & 40 & 0 & 95 & 98 & 218 & 0 & 0 & 11.29 & 33 \\
\hline & a- $0 \downarrow 0$ & 40 & 0 & 0 & 135 & 98 & 558 & 0 & 0 & 23.43 & 91 \\
\hline \multirow[t]{4}{*}{ init_vars } & alf- $0 \downarrow 0$ & 137 & 72 & 5 & 0 & 67 & 5 & 0 & 0 & 2.88 & 7 \\
\hline & al- $0 \downarrow 0$ & 137 & 0 & 77 & 0 & 67 & 5 & 0 & 0 & 2.88 & 7 \\
\hline & af $-0 \downarrow 0$ & 137 & 72 & 0 & 5 & 67 & 5 & 0 & 0 & 2.88 & 7 \\
\hline & a- $0 \downarrow 0$ & 137 & 0 & 0 & 77 & 67 & 5 & 0 & 0 & 2.88 & 7 \\
\hline \multirow[t]{4}{*}{ mapcolor' } & alf- $0 \downarrow 0$ & 11 & 13 & 1 & 58 & 42 & 101 & 0 & 0 & 6.81 & 25 \\
\hline & al $-0 \downarrow 0$ & 11 & 0 & 14 & 58 & 42 & 101 & 0 & 0 & 6.81 & 25 \\
\hline & af- $0 \downarrow 0$ & 11 & 13 & 0 & 59 & 42 & 101 & 0 & 0 & 6.81 & 25 \\
\hline & a- $0 \downarrow 0$ & 11 & 0 & 0 & 72 & 42 & 165 & 0 & 0 & 9.86 & 63 \\
\hline \multirow[t]{4}{*}{ mapcolor } & alf- $0 \downarrow 0$ & 52 & 25 & 6 & 10 & 33 & 10 & 0 & 0 & 3.31 & 10 \\
\hline & al $-0 \downarrow 0$ & 52 & 0 & 31 & 10 & 33 & 10 & 0 & 0 & 3.31 & 10 \\
\hline & af $-0 \downarrow 0$ & 52 & 25 & 0 & 16 & 33 & 13 & 0 & 0 & 3.54 & 10 \\
\hline & a- $0 \downarrow 0$ & 52 & 0 & 0 & 41 & 33 & 1083 & 0 & 0 & 85.85 & 1023 \\
\hline
\end{tabular}

Table 4: Impact of annotation lattice on precision 


\begin{tabular}{|c|c|c|c|c|c|c|c|c|c|c|c|}
\hline Program & Ann-k & $\mathrm{Gr}$ & $\mathrm{Fr}$ & Lin & Any & Sin & NSin & Str & Sval & $\mathrm{AvS}$ & $\mathrm{MxS}$ \\
\hline \multirow[t]{4}{*}{ grammar } & 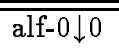 & 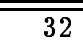 & $\overline{\overline{13}}$ & $\overline{7} 7$ & $\overline{0}$ & $\overline{20}$ & 0 & $\overline{0}$ & 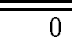 & 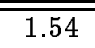 & 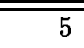 \\
\hline & al- $0 \downarrow 0$ & 32 & 0 & 20 & 0 & 20 & 0 & 0 & 0 & 1.54 & 5 \\
\hline & $a f-0 \downarrow 0$ & 32 & 13 & 0 & 7 & 20 & 0 & 0 & 0 & 1.54 & 5 \\
\hline & $a-0 \downarrow 0$ & 32 & 0 & 0 & 20 & 20 & 0 & 0 & 0 & 1.54 & 5 \\
\hline \multirow[t]{4}{*}{ browse } & alf- $0 \downarrow 0$ & 214 & 172 & 103 & 79 & 279 & 570 & 0 & 0 & 9.23 & 512 \\
\hline & al- $0 \downarrow 0$ & 214 & 0 & 275 & 79 & 279 & 570 & 0 & 0 & 9.23 & 512 \\
\hline & $a f-0 \downarrow 0$ & 214 & 172 & 0 & 182 & 279 & 670 & 0 & 0 & 10.32 & 512 \\
\hline & $\mathrm{a}-0 \downarrow 0$ & 214 & 0 & 0 & 354 & 279 & 1217 & 0 & 0 & 16.26 & 512 \\
\hline \multirow[t]{4}{*}{ browse_u } & alf- $0 \downarrow 0$ & 176 & 125 & 97 & 76 & 245 & 551 & 0 & 0 & 9.26 & 512 \\
\hline & al- $0 \downarrow 0$ & 176 & 0 & 222 & 76 & 245 & 551 & 0 & 0 & 9.26 & 512 \\
\hline & $a f-0 \downarrow 0$ & 176 & 125 & 0 & 173 & 245 & 650 & 0 & 0 & 10.41 & 512 \\
\hline & $\mathrm{a}-0 \downarrow 0$ & 176 & 0 & 0 & 298 & 245 & 693 & 0 & 0 & 10.91 & 512 \\
\hline \multirow[t]{4}{*}{ bid } & $\overline{\text { alf- } 0 \downarrow 0}$ & 340 & 123 & 1 & 0 & 124 & 0 & 0 & 0 & 2.25 & 8 \\
\hline & al- $0 \downarrow 0$ & 340 & 0 & 124 & 0 & 124 & 0 & 0 & 0 & 2.25 & 8 \\
\hline & af $-0 \downarrow 0$ & 340 & 123 & 0 & 1 & 124 & 0 & 0 & 0 & 2.25 & 8 \\
\hline & $\mathrm{a}-0 \downarrow 0$ & 340 & 0 & 0 & 124 & 124 & 34 & 0 & 0 & 2.87 & 19 \\
\hline \multirow[t]{4}{*}{ rdtok } & alf- $0 \downarrow 0$ & 337 & 320 & 145 & 43 & 498 & 46 & 0 & 0 & 2.86 & 7 \\
\hline & al- $0 \downarrow 0$ & 337 & 0 & 465 & 43 & 498 & 46 & 0 & 0 & 2.86 & 7 \\
\hline & af $-0 \downarrow 0$ & 337 & 320 & 0 & 188 & 498 & 50 & 0 & 0 & 2.88 & 7 \\
\hline & $\mathrm{a}-0 \downarrow 0$ & 337 & 0 & 0 & 508 & 498 & 271 & 0 & 0 & 4.05 & 17 \\
\hline \multirow[t]{4}{*}{ deriv } & alf- $0 \downarrow 0$ & 523 & 193 & 0 & 0 & 193 & 0 & 0 & 0 & 1.74 & 4 \\
\hline & al- $0 \downarrow 0$ & 523 & 0 & 193 & 0 & 193 & 0 & 0 & 0 & 1.74 & 4 \\
\hline & af $-0 \downarrow 0$ & 523 & 193 & 0 & 0 & 193 & 0 & 0 & 0 & 1.74 & 4 \\
\hline & $\mathrm{a}-0 \downarrow 0$ & 523 & 0 & 0 & 193 & 193 & 0 & 0 & 0 & 1.74 & 4 \\
\hline \multirow[t]{4}{*}{ kalah } & alf- $0 \downarrow 0$ & 969 & 238 & 28 & 5 & 271 & 1 & 0 & 0 & 1.97 & 7 \\
\hline & al- $0 \downarrow 0$ & 969 & 0 & 266 & 5 & 271 & 1 & 0 & 0 & 1.97 & 7 \\
\hline & af $-0 \downarrow 0$ & 969 & 238 & 0 & 33 & 271 & 2 & 0 & 0 & 1.98 & 7 \\
\hline & a- $0 \downarrow 0$ & 969 & 0 & 0 & 271 & 271 & 10 & 0 & 0 & 2.04 & 7 \\
\hline \multirow[t]{4}{*}{ tarjan } & alf- $0 \downarrow 0$ & 1037 & 449 & 120 & 301 & 817 & 346 & 0 & 0 & 4.31 & 35 \\
\hline & al- $0 \downarrow 0$ & 1037 & 0 & 569 & 301 & 817 & 346 & 0 & 0 & 4.31 & 35 \\
\hline & af $-0 \downarrow 0$ & 1037 & 449 & 0 & 421 & 817 & 392 & 0 & 0 & 4.48 & 35 \\
\hline & $a-0 \downarrow 0$ & 1037 & 0 & 0 & 870 & 817 & 680 & 0 & 0 & 5.54 & 68 \\
\hline \multirow[t]{4}{*}{ read } & alf- $0 \downarrow 0$ & 1370 & 709 & 4 & 4 & 715 & 2 & 0 & 0 & 3.32 & 8 \\
\hline & al- $0 \downarrow 0$ & 1370 & 0 & 713 & 4 & 715 & 2 & 0 & 0 & 3.32 & 8 \\
\hline & af $-0 \downarrow 0$ & 1370 & 709 & 0 & 8 & 715 & 2 & 0 & 0 & 3.32 & 8 \\
\hline & $\mathrm{a}-0 \downarrow 0$ & 1370 & 0 & 0 & 717 & 715 & 17 & 0 & 0 & 3.39 & 8 \\
\hline \multirow[t]{4}{*}{ ch_db_gr } & alf- $0 \downarrow 0$ & 1106 & 212 & 29 & 20 & 259 & 16 & 0 & 0 & 2.31 & 8 \\
\hline & al- $0 \downarrow 0$ & 1106 & 0 & 241 & 20 & 259 & 16 & 0 & 0 & 2.31 & 8 \\
\hline & af $-0 \downarrow 0$ & 1106 & 212 & 0 & 49 & 259 & 16 & 0 & 0 & 2.31 & 8 \\
\hline & $\mathrm{a}-0 \downarrow 0$ & 1106 & 0 & 0 & 261 & 259 & 40 & 0 & 0 & 2.51 & 9 \\
\hline \multirow[t]{4}{*}{ ch_db } & alf- $0 \downarrow 0$ & 910 & 217 & 214 & 35 & 438 & 42 & 0 & 0 & 2.67 & 11 \\
\hline & al- $0 \downarrow 0$ & 910 & 0 & 431 & 35 & 438 & 42 & 0 & 0 & 2.67 & 11 \\
\hline & $a f-0 \downarrow 0$ & 910 & 217 & 0 & 249 & 438 & 686 & 0 & 0 & 6.24 & 63 \\
\hline & $a-0 \downarrow 0$ & 910 & 0 & 0 & 466 & 438 & 1002 & 0 & 0 & 8.00 & 127 \\
\hline \multirow[t]{4}{*}{ peephole } & alf- $0 \downarrow 0$ & 593 & 205 & 120 & 186 & 366 & 458 & 0 & 0 & 3.94 & 33 \\
\hline & al- $0 \downarrow 0$ & 593 & 0 & 325 & 186 & 366 & 458 & 0 & 0 & 3.94 & 33 \\
\hline & af $-0 \downarrow 0$ & 593 & 205 & 0 & 306 & 366 & 472 & 0 & 0 & 4.01 & 33 \\
\hline & $a-0 \downarrow 0$ & 593 & 0 & 0 & 511 & 366 & 740 & 0 & 0 & 5.29 & 64 \\
\hline \multirow[t]{4}{*}{ boyer } & alf- $0 \downarrow 0$ & 137 & 66 & 447 & 48 & 466 & 120 & 0 & 0 & 2.82 & 18 \\
\hline & al- $0 \downarrow 0$ & 137 & 0 & 513 & 48 & 466 & 120 & 0 & 0 & 2.82 & 18 \\
\hline & af $-0 \downarrow 0$ & 137 & 66 & 0 & 495 & 466 & 848 & 0 & 0 & 6.32 & 127 \\
\hline & $\mathrm{a}-0 \downarrow 0$ & 137 & 0 & 0 & 561 & 466 & 890 & 0 & 0 & 6.52 & 127 \\
\hline \multirow[t]{4}{*}{ icomp } & alf- $0 \downarrow 0$ & 816 & 1032 & 131 & 1419 & 1927 & 2504 & 0 & 0 & 7.87 & 57 \\
\hline & al- $0 \downarrow 0$ & 816 & 0 & 1163 & 1419 & 1927 & 2504 & 0 & 0 & 7.87 & 57 \\
\hline & af $-0 \downarrow 0$ & 816 & 1032 & 0 & 1550 & 1927 & 2504 & 0 & 0 & 7.87 & 57 \\
\hline & $a-0 \downarrow 0$ & 816 & 0 & 0 & 2582 & 1927 & 3676 & 0 & 0 & 9.95 & 81 \\
\hline
\end{tabular}

Table 4: Impact of annotation lattice on precision (continued) 
AvS: Average size of sharing components.

MxS: Maximum size of sharing components.

The information shown in the last four columns concerns in fact the values before projection onto the program variables. Compared with the analysis for depth 2, the depth 3 analysis only improves the precision of the sharing component for the mapcolor' benchmark (94 instead of 101 non-singleton sharing groups).

Table 4 is similar, but compares the precision results at depth 0 for the four annotation lattices $\{a, l, f\},\{a, l\},\{a, f\}$, and $\{a\}$. Note that identical groundness information is derived for all lattices. The sum of variables with mode free, linear, and any is also constant. The differences are in the amount of possible sharing information that is derived, both absolute number of sharing groups and average size of sharing components. The same effect holds for other depths; as an illustration, the corresponding table for depth 2 is in Appendix D, where one can see that the same amount of structural and sub-term equality information is derived for all four lattices.

\subsection{Discussion}

There is no simple relation between the analysis time and the depth $k$ of the analyses (see Table 1, first part). The factors influencing the analysis time are:

- The presence of structural information which can substantially increase the number of call patterns, and consequently the analysis time. This effect is very clear in the benchmarks mapcolor', mapcolor, tarjan, read, and boyer.

The number of call patterns is an indication of the amount of work that has to be done during the analysis. Other indications of the amount of work are the number of iterations, which can be larger for greater depths even for the same number of call patterns (e.g., for the init_vars, rdtok, and peephole benchmarks) or the number of unifications (both numbers are given in the tables of Appendix D).

- The presence of structural information which can increase the number of abstract variables and thus also the potential size of sharing sets. This effect is very clear in the benchmark mapcolor' where a steep increase in the average size of the sharing components (see Table 3) causes a sharp increase of the analysis time. For the mapcolor, ch_db, and icomp benchmarks, the effect is less clear-cut and the increased analysis time is also due to a combination of increased number of call patterns and iterations/unifications.

On the other hand, the structural information sometimes enhances precision, resulting in smaller sharing components. This happens in the browse, browse_u, and the peephole benchmarks when increasing depth 1 to depth 2, making the analysis faster than the depth 0 analysis.

The relation between the analysis time and the annotation lattice in the analyses (see Table 1, second part), can be summarized as follows:

- The $\{a, l, f\}$ and $\{a, l\}$ analyses are comparable in execution time except for the rdtok benchmark where the number of iterations/unifications makes the difference. 
- The $\{a, f\}$ and $\{a, l\}$ analyses are also comparable in execution time except for the ch_db and boyer benchmarks for which linearity information appears to be more interesting than freeness information. The lack of linearity information leads to an increased average size of sharing components for the serialize $(4.39 \rightarrow 11.29)$, ch_db $(2.67 \rightarrow 6.24)$, and boyer $(2.82 \rightarrow 6.32)$ benchmarks.

- The performance of the $\{a\}$ analysis is much worse for nine of the nineteen benchmark programs; the increase in execution time may be due to increased average size for the sharing component (this is the case for serialize, mapcolor, browse, tarjan, ch_db, peephole, boyer, icomp, and ann) and/or to an increased number of call-success patterns (for serialize, browse, browse_u, peephole, and ann).

(Note that for the a-0 analysis, both the annotation mapping and the equation component are in fact overhead since a distinct abstract variable is associated with each concrete program variable.)

- For programs with the same number of call patterns and iterations for all four lattices (namely mapcolor, grammar, bid, deriv, and ch_db_gr), there are only very small differences in the analysis time, except for the a- 0 analysis of mapcolor (due to a very high average size of the sharing components; Table 4).

The size of the abstract equation systems showing up during the analysis depends on the depth $k$ chosen, the maximal arity $a_{\max }$ of function symbols occurring in the benchmark programs, and the maximal number $c v_{\max }$ of concrete variables in the clauses of these programs. The maximal number of abstract variables occurring in an abstract equation system is bounded by $a v_{\max }=c v_{\max } * a_{\max }^{k}$, which is highly program dependent. The size of the sharing sets can be a problem; it may be as large as $2^{a v_{\max }}-1$. This happens for instance in one program point of the mapcolor benchmark in the case of the a- 0 parameter setting. The map-representation fact contains 10 concrete variables, all of which have mode any and all possibly share with one another. Consequently, a sharing component consisting of 1023 sharing groups is derived (see $\mathrm{MxS}$ column in Table 4). It can cause the analysis time to be unacceptably large, even make the analysis impossible as in the icomp benchmark for depth 3 and the ann benchmark for depths greater than 1 .

Comparing the analysis time of the $\{a, l, f\}$ domain for $k=0$ with the timings obtained for the $P \star S F$ domain in [13] that encodes similar information, we see that the abstract equation system is about two times slower. Since our system is a first prototype, instrumented to gather information about the analysis' behavior, and since the particular domain $\mathcal{A} E q s(0,\{a, l, f\})$ does not fully exploit the generality of the system (it has no structural information), we conclude that the abstract equation system is a viable tool for experimenting with a broad range of parameter settings.

Table 2 shows that groundness is a very important special case; the groundness rule of Algorithm 3 is activated in more than half of all cases. Although the freeness rule is often activated (in the $\{a, l, f\}$ and $\{a, f\}$ lattice domains) and is essential to obtain freeness information, for what concerns all other information, the $\{a, l\}$ annotation domain gives exactly the same information as the $\{a, l, f\}$ annotation domain when comparing the least upper-bound of all specializations (see Table 4). In a few benchmarks, the $\{a, l, f\}$ annotation domain has some extra call patterns (so the analysis takes more time). The extra call patterns derived have more precise annotations ( $f$ instead of $l$ ); there is no difference in the sharing information, as one could expect since the optimization of the sharing computation based on freeness, resp. linearity, is the same. The $\{a, f\}$ 
annotation domain, however, while giving the same groundness and freeness information as the $\{a, l, f\}$ annotation domain, gives for several benchmarks (serialize, browse, browse_u, ch_db, and boyer) much worse sharing information (see again Table 4). In [13], the linearity encoded in the $P$ domain [38] increased the precision of set-sharing in the reduced product domain for the same benchmarks. For the $\{a\}$ annotation domain, the sharing information derived further deteriorates as one would expect.

Table 3 shows that the inclusion of structural information substantially enhances the precision only for the benchmarks browse, browse_u, and peephole. Comparison with the $0^{*}$ analysis indicates that the increased precision is mainly due to passing structural information between call and head. We observed that not only the depth $0^{*}$ analysis hardly shows any improvement compared with the depth 0 analysis, but neither the depth 1 analysis does. Moreover, a few experiments with fully normalized programs also showed no difference. All these observations confirm that the set-sharing is very good at abstracting the information present in the structure component which is relevant for the precision of the mode and the sharing information. This is contrary to the situation of pair-sharing for which Van Hentenryck and Le Charlier [15, 30,31] introduced the pattern domain to recover the information lost due to the normalization of programs.

Consider once more the number of specialized versions (or call patterns) analyzed for each predicate (Table 1). For most benchmarks, this number increases with the depth parameter of the analysis ${ }^{2}$. The number of versions can become quite large and therefore has a great impact on the analysis time (e.g., for the 36 predicates of the tarjan benchmark, 70 specialized versions are derived for depth 0 and as many as 314 versions for depth 3 ). In our experiments, we reduced the number of versions being analyzed by semi-normalizing some of the programs (e.g., compare the benchmarks browse_u (unnormalized) and browse (semi-normalized) in Table 1). However, it is most likely that different versions eventually lead to the same compiler optimizations. For instance, we observed that different call patterns for a predicate often contain the same mode information for the arguments, except in the case of basic predicates such as for example append/3. A dynamic control of the versions that are analyzed therefore seems desirable, especially for the kind of abstractions discussed in the present paper. In order to study this aspect, we would like to have an abstract interpretation system that decides dynamically which specialized call patterns are analyzed. The decision could be based on heuristic information about the optimization possibilities. For the abstractions considered in this paper, we could use the following heuristic: analyze a new specialized call pattern if it has an interesting difference in mode information from previous versions analyzed or if one of the (partially) instantiated arguments does not match with all the clauses for the predicate. The latter condition is related to information used for the choice of indexing arguments. Further work consists in adapting the abstract interpretation system for such dynamic control and in tuning appropriate heuristics.

\section{Related Work}

Apart from the abstract domains mentioned in the introduction, several other domains for variable sharing, linearity, and freeness information have been proposed in the past, e.g. $[14,27,28,40]$. It is outside the present scope to discuss all of these in detail. Also

\footnotetext{
${ }^{2}$ Only for the peephole benchmark there is a decrease due to the enhanced precision for depths greater than 1 .
} 
Getzinger [20] studied the cost and benefits of a collection of mode, type, and sharing domains integrated in a compiler. Our work is different in that we use a single parametric domain that can be instantiated to several of the domains considered promising by Getzinger and we trace in greater detail the impact of various domain properties and the optimization rules that they permit. On the other hand, since our analyzer is not integrated into a compiler, we were not able to measure the impact of the analysis on the runtime of benchmark programs and compare this aspect with Getzinger's findings.

The abstract equation system is related to the generic pattern domain, Pat $(\mathcal{R})$, of $[15$, 30,31 . The abstract equations component is an alternative representation of the pattern component of Pat $(\mathcal{R})$. The latter provides both structural and same-value information but uses integer indices to identify subterms instead of abstract variables. The advantage of using abstract variables is that the algorithms can be formulated at a higher level. The sharing component and annotation mapping can be seen as a particular instance of the generic part $\mathcal{R}$ for associating information with the so-called leaves (subterms for which the principal functor is not known). Information on non-leaf subterms is easily derived recursively from the information on the leaves; for the application at hand, there is no need to associate any additional information with non-leaf subterms. The instance of the generic part in the present work is more expressive than the one used in [15] which did not represent the linearity property.

In [15], Cortesi et al. presented a formalism, the open product construct, for combining automatically existing domains which can gain precision by interacting with one another. The domain of abstract equation systems presented here also combines different components that interact in order to improve the precision. It was developed independently from [15], and in retrospect, it would not have been possible to express the application within the formalism of open products for simplifying the correctness proofs and still obtain the same degree of precision. In the terminology of [15], predicates such as share AEqs $_{\text {and }}$ free $_{\mathrm{AEqs}}$ are queries, i.e. boolean functions providing information to the 'environment', which is then available to the operations of each domain involved in the combination. Operations can interact in terms of the abstract objects before or after execution of the operation. The unification operation for the abstract equation systems on the other hand permits interaction in terms of abstract objects at intermediate stages too. Moreover, the soundness results for the automatable open product presented in [15] only address a small class of queries that are disjunctions of queries on the domains combined. They are not readily applicable for more general communication patterns. As an example, consider the linear $_{A E q s}$ predicate which tells whether an abstract term is linear or not. If the abstract term is simply an abstract variable, then a query to the annotation component suffices. However, for other abstract terms, linearity can neither be formulated as a query to a single component of AEqs nor as a disjunction of such simple queries. Indeed, linearity is specified as follows:

$$
\begin{aligned}
& \operatorname{linear}_{\mathrm{AEqs}}\left(\tau\left[@_{1}, \ldots, @_{n}\right]\right) \Leftrightarrow \forall i \in\{1, \ldots, n\}: a n n_{\mathrm{AEqs}}\left(@_{i}\right) \leq l \& \\
& \neg\left(\exists i \in\{1, \ldots, n\}:\left(@_{i}\right. \text { occurs more than once in }\right. \\
&\left.\left.\tau\left[@_{1}, \ldots, @_{n}\right] \& @_{i} \in \operatorname{AVar}(\Delta)\right)\right) \& \\
& \neg\left(\exists i, j \in\{1, \ldots, n\}:\left(i \neq j \& \exists \in \in \Delta .\left\{@_{i}, @_{j}\right\} \subseteq S\right)\right) .
\end{aligned}
$$

In [15], the authors mention in their conclusion that viewing all operations as coroutines communicating information whenever appropriate might be a promising generalization of the open product construct. But as far as we know, no soundness results for such a fully automatable generalized formalism have been presented yet. 


\section{Conclusion}

Abstract equation systems have been introduced [10] to allow a simple and intuitive correctness proof of abstract unification - showing that the abstract algorithm mimics a well-chosen concrete one. In this paper we presented a variant - substantially improving upon [4] - that preserves this property and at the same time is suited as a basis for an implementation. The resulting system is very general. It can be parameterized with an annotation mapping, a depth, and a sharing component. The implementation uses the generic abstract interpretation system PLAI [22, 35, 36, 37]. For a particular instantiation not exploiting the presence of structural information, timings have the same order of magnitude as those reported for a particular domain, $P \star S F$ [13], encoding similar information.

Structural information (at depth 2) turned out to improve the precision of the mode and sharing information only in three out of nineteen benchmarks. In those cases, the improved precision originated from passing structural information between call and head. Precision was unaffected by semi-normalization and normalization, though such program transformations can substantially influence the analysis time. Sporadic, analysis time becomes unacceptably large due to the presence of very large sharing sets. This is a drawback of the set-sharing representation. Since structural information is by itself relevant information for an optimizing compiler and the variable dependencies present in the structural information seem to be redundantly present in the set-sharing component, replacing set-sharing by pair-sharing may provide a better trade-off between performance and precision.

Our findings confirm that linearity is important and can significantly improve the precision of set-sharing. This was already observed in [13] where the linearity, encoded in the $P$ domain, improved the precision of the reduced product domains $P \star S$ and $P \star S F$.

Another way to improve performance would be to dynamically control the number of different call patterns that are analyzed for a predicate. If the difference between two call patterns is not significant w.r.t. the properties of interest to the compiler, then it is probably more economical to analyze the call pattern that is the least upper-bound of both patterns. However, current generic abstract interpretation systems such as PLAI [37] and GAIA [31] do not allow any dynamic control.

\section{Acknowledgement}

We are grateful to Manuel Hermenegildo, Maria García de la Banda, and the other developers and implementors of the PLAI system for abstract interpretation at UPM-CLIP for making their system and set of benchmark programs available to us, and to Bart Demoen for the discussion on implementation techniques and compiler indexing heuristics.

\section{References}

[1] J. Almgren, S. Andersson, M. Carlsson, L. Flood, S. Haridi, C. Frisk, H. Nilsson, and J. Sundberg. SICStus Prolog Library Manual. Swedish Institute of Computer Science, Jan. 1993.

[2] R. Barbuti, R. Giacobazzi, and G. Levi. A general framework for semantics-based bottom-up abstract interpretation of logic programs. ACM Trans. Prog. Lang. Syst., 15(1):133-181, Jan. 1993. 
[3] M. Bruynooghe. A practical framework for the abstract interpretation of logic programs. The Journal of Logic Programming, 10(2):91-124, 1991.

[4] M. Bruynooghe and M. Codish. Freeness, sharing, linearity and correctness - all at once. In P. Cousot, M. Falaschi, G. Filè, and A. Rauzy, editors, Proceedings of the Third International Workshop on Static Analysis, LNCS 724, pages 153-164, Padova, Italy, Sept. 1993. SpringerVerlag.

[5] M. Bruynooghe, M. Codish, and A. Mulkers. Abstract unification for a composite domain deriving sharing and freeness properties of program variables. In F. S. de Boer and M. Gabbrielli, editors, Proceedings of the W2 Post-conference Workshop on Verification and Analysis of Logic Languages, ICLP94, pages 213-229, June 1994.

[6] M. Bruynooghe, M. Codish, and A. Mulkers. A composite domain for freeness, sharing, and compoundness analysis of logic programs. Report CW 196, Dept. of Computer Science, K.U.Leuven, July 1994. Available via anonymous ftp on request.

[7] M. Bruynooghe, G. Janssens, A. Callebaut, and B. Demoen. Abstract interpretation: Towards the global optimization of Prolog programs. In Proceedings of the Fifth International Conference and Symposium on Logic Programming, pages 192-204, San Francisco, August September 1987. IEEE, Computer Society Press.

[8] F. Bueno, M. García de la Banda, and M. Hermenegildo. Effectiveness of global analysis in strict independence-based automatic parallelization. In M. Bruynooghe, editor, Proceedings of the International Symposium on Logic Programming, pages 320-336. MIT Press, Nov. 1994.

[9] M. Carlsson, J. Widén, et al. SICStus Prolog User's Manual. Swedish Institute of Computer Science, Jan. 1993.

[10] M. Codish, D. Dams, G. Filé, and M. Bruynooghe. Freeness analysis for logic programs - and correctness? In D. S. Warren, editor, Proceedings of the Tenth International Conference on Logic Programming, pages 116-131, Budapest, Hungary, 1993. The MIT Press. An extended version is available as Technical Report, Dept. of Comp. Science, K.U.Leuven.

[11] M. Codish, D. Dams, and E. Yardeni. Derivation and safety of an abstract unification algorithm for groundness and aliasing analysis. In K. Furukawa, editor, Proceedings of the Eighth International Conference on Logic Programming, pages 79-93, Paris, France, 1991. The MIT Press, Cambridge, Mass.

[12] M. Codish, D. Dams, and E. Yardeni. Bottom-up abstract interpretation of logic programs. Theoretical Computer Science, 124(1):93-126, Feb. 1994.

[13] M. Codish, A. Mulkers, M. Bruynooghe, M. García de la Banda, and M. Hermenegildo. Improving abstract interpretations by combining domains. In Proceedings of PEPM'93, the ACM SIGPLAN Symposium on Partial Evaluation and Semantics-Based Program Manipulation, pages 194-205, Copenhagen, June 1993. Revised version to appear in ACM Trans. Prog. Lang. Syst.

[14] A. Cortesi and G. Filé. Abstract interpretation of logic programs: An abstract domain for groundness, sharing, freeness and compoundness analysis. In P. Hudak and N. D. Jones, editors, Proceedings of PEPM'91, the ACM SIGPLAN Symposium on Partial Evaluation and Semantics-Based Program Manipulation, pages 52-61, 1991.

[15] A. Cortesi, B. Le Charlier, and P. Van Hentenryck. Combinations of abstract domains for logic programming. In Conference Record of POPL'94: 21st ACM SIGPLAN-SIGACT Symposium on Principles of Programming Languages, pages 227-239, Portland, Oregon, 1994. ACM Press.

[16] P. Cousot and R. Cousot. Abstract interpretation and application to logic programs. The Journal of Logic Programming, 13(2 and 3):103-179, 1992. 
[17] V. Dumortier, G. Janssens, M. Bruynooghe, and M. Codish. Freeness analysis in the presence of numerical constraints. In Tenth International Conference on Logic Programming, pages 100-115. MIT Press, June 1993.

[18] M. García de la Banda and M. Hermenegildo. A practical approach to the global analysis of constraint logic programs. In 1993 International Logic Programming Symposium, pages 437-455. MIT Press, Cambridge, MA, October 1993.

[19] M. J. García de la Banda García. Independence, Global Analysis, and Parallelism in Dynamically Scheduled Constraint Logic Programming. PhD thesis, Universidad Politécnica de Madrid (UPM), July 1994.

[20] T. W. Getzinger. The costs and benefits of abstract interpretation-driven Prolog optimization. In B. Le Charlier, editor, Proceedings of the First International Static Analysis Symposium, SAS'94, Lecture Notes in Computer Science 864, pages 1-25. Springer-Verlag, 1994.

[21] M. Hermenegildo and K. J. Greene. \&-Prolog and its performance: Exploiting independent and-parallellism. In D. H. D. Warren and P. Szeredi, editors, Proceedings of the Seventh International Conference on Logic Programming, pages 253-268, Jerusalem, 1990. MIT Press, Cambridge.

[22] M. Hermenegildo, R. Warren, and S. Debray. Global flow analysis as a practical compilation tool. Journal of Logic Programming, 13(4):349-367, August 1992.

[23] D. Jacobs and A. Langen. Accurate and efficient approximation of variable aliasing in logic programs. In E. L. Lusk and R. A. Overbeek, editors, Proceedings of the North American Conference on Logic Programming, pages 154-165, Cleveland, Ohio, USA, 1989.

[24] D. Jacobs and A. Langen. Static analysis of logic programs for independent AND-parallelism. The Journal of Logic Programming, 13(2 and 3):291-314, 1992.

[25] G. Janssens and M. Bruynooghe. Deriving descriptions of possible values of program variables by means of abstract interpretation. The Journal of Logic Programming, 13 (2 and 3):205-258, 1992.

[26] N. D. Jones and H. Søndergaard. A semantic-based framework for the abstract interpretation of Prolog. In S. Abramsky and C. Hankin, editors, Abstract Interpretation of Declarative Languages, Ellis Horwood Series in Computers and their Applications, pages 123-142. Ellis Horwood, Chichester, 1987.

[27] A. King. A synergistic analysis for sharing and groundness which traces linearity. In D. Sannella, editor, Proceedings of Fifth European Symposium on Programming Languages and Systems - ESOP'94, pages 363-378, Edinburgh, 1994. Springer-Verlag.

[28] A. King and P. Soper. Depth- $k$ sharing and freeness. In P. Van Hentenryck, editor, Proceedings of the 11th International Conference on Logic Programming, pages 553-568, Santa Margherita Ligure, Italy, 1994.

[29] J.-L. Lassez, M. J. Maher, and K. Marriott. Unification revisited. In J. Minker, editor, Foundations of Deductive Databases and Logic Programming, pages 587-625. Morgan Kaufmann Publishers Inc., Los Altos, 1988.

[30] B. Le Charlier and P. Van Hentenryck. Reexecution in abstract interpretation of Prolog. In K. Apt, editor, Proceedings of the Joint International Conference and Symposium on Logic Programming, pages 750-764, Washington D.C., 1992. MIT Press, Cambridge.

[31] B. Le Charlier and P. Van Hentenryck. Experimental evaluation of a generic abstract interpretation algorithm for Prolog. ACM Trans. Prog. Lang. Syst., 16(1):35-101, Jan. 1994.

[32] K. Marriott. Frameworks for abstract interpretation. Acta Informatica, 30(2):103-129, 1993.

[33] A. Martelli and U. Montanari. An efficient unification algorithm. ACM Transactions on Programming Languages and Systems, 4(2):258-282, Apr. 1982. 
[34] K. Muthukumar and M. Hermenegildo. Determination of variable dependence information through abstract interpretation. In E. L. Lusk and R. A. Overbeek, editors, Proceedings of the North American Conference on Logic Programming, pages 166-188, Cleveland, Ohio, USA, 1989.

[35] K. Muthukumar and M. Hermenegildo. Deriving a fixpoint computation algorithm for topdown abstract interpretation of logic programs. Technical Report ACT-DC-153-90, Microelectronics and Computer Technology Corporation (MCC), Austin, TX 78759, April 1990.

[36] K. Muthukumar and M. Hermenegildo. Combined determination of sharing and freeness of program variables through abstract interpretation. In K. Furukawa, editor, Proceedings of the Eighth International Conference on Logic Programming, pages 49-63, Paris, France, 1991. The MIT Press.

[37] K. Muthukumar and M. Hermenegildo. Compile-time derivation of variable dependency using abstract interpretation. The Journal of Logic Programming, 13(2 and 3):315-347, 1992.

[38] H. Søndergaard. An application of abstract interpretation of logic programs: Occur check reduction. In B. Robinet and R. Wilhelm, editors, ESOP'86 Proceedings European Symposium on Programming, Lecture Notes in Computer Science 213, pages 327-338. Springer-Verlag, N.Y., 1986.

[39] L. Sterling and E. Shapiro. The Art of Prolog: Advanced Programming Techniques. Logic Programming Series. MIT Press, second edition, Apr. 1994.

[40] R. Sundararajan and J. Conery. An abstract interpretation scheme for groundness, freeness and sharing analysis of logic programs. In Conference on Foundations of Software Technology and Theoretical Computer Science, number 652 in Lecture Notes in Computer Science, pages 203-216. New Delhi, Springer-Verlag, December 1992.

[41] P. Van Hentenryck, A. Cortesi, and B. Le Charlier. Type analysis of Prolog using type graphs. Technical Report CS-93-52, Brown University, Department of Computer Science, Nov. 1993. To appear in Journal of Logic Programming. 


\section{Appendix}

\section{A Some Implementation Details}

In this section, we briefly describe the data structures used to represent abstract equation systems in the analyzer. Abstract variables in mixed terms are represented by the special function symbol '@'/1; the argument specifies the number of the abstract variable. The equations component is represented as a list of equations. The sharing groups of abstract variables are encoded as integers (note: the range of integers in SICStus Prolog can be considered infinite [9]). The sharing component is implemented as a list of such bit-encoded sharing groups. The annotations component is implemented using the SICStus Prolog library module for extendible arrays with logarithmic access time [1]. Highly numbered abstract variables are more costly to represent, both in the sharing component and in the annotation mapping. Consistent renaming of the abstract variables reduces this cost. An alternative data structure for the annotations component consists of two bit-encoded sets representing respectively the set of free and linear abstract variables. The former representation is more suited for future extensions of the annotation set.

Example A.1 Consider the set of concrete variables $V=\{A, B, C, D, E\}$ and the $a b$ stract equation system

$$
\mathrm{AEqs}_{V}=\left\{\left\{\begin{array}{l}
A=@_{1}^{f}, B=f\left(@_{7}^{l}, g\left(@_{1}^{f}\right), @_{1}^{f}, @_{3}^{a}\right), \\
C=@_{5}^{f}, D=@_{4}^{l}, E=f\left(@_{4}^{l}\right)
\end{array}\right\} ;\left\{\begin{array}{l}
\left\{@_{1}^{f}\right\},\left\{@_{3}^{a}\right\}, \\
\left\{@_{1}^{f}, @_{4}^{l}, @_{5}^{f}\right\},\left\{@_{5}^{f}\right\}
\end{array}\right\}\right\rangle .
$$

The internal representation of $\mathrm{AEqs}_{V}$ is:

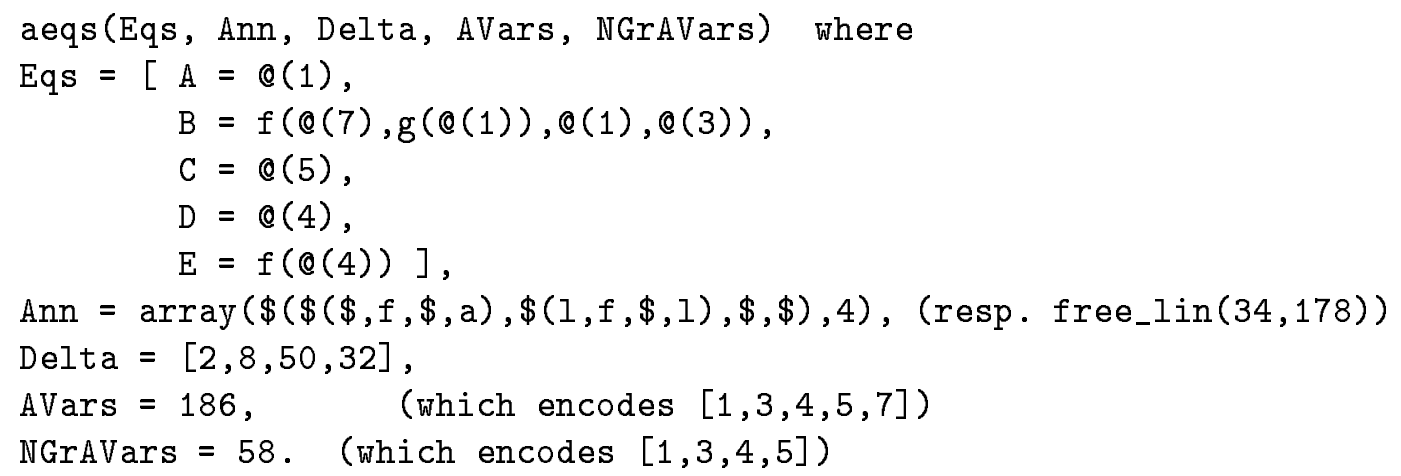

(Note: for efficient basic operations, two additional components are included, namely the set of abstract variables of the equation system, AVars, and the subset of possibly nonground abstract variables, NGrAVars.)

\section{B The Benchmarks}

The benchmarks include several programs used previously in the evaluation of related abstract domains $[13,15,20,41]$. They may contain if-then-else constructs, or-clauses, and calls for the 'not/1' or 'findall/3' metacalls, but no dynamic predicates changable by assert or retract.

The first program, serialize, is a program manipulating lists of numbers; init_vars initializes two abstract substitutions to have the same set of variables; mapcolor is a 
map coloring program for two map representations of respectively six and ten countries; mapcolor' is the same program but with the order of some subgoals of the main procedure switched in order to have groundness propagation from right to left instead of vice versa and only considering the map representation for six countries; grammar is a program which generates and recognizes a small set of English; browse_u is a program for pattern matching taken from the Gabriel benchmarks (as translated by T. Dobry and H. Touati); browse is the same program but with all subgoals semi-normalized; bid computes an opening bid for a bridge hand; rdtok is O'Keefe's public domain Prolog tokenizer; deriv performs symbolic differentiation of an equation; kalah is the Kalah playing program from [39] which uses alpha-beta pruning; tarjan is a program for computing strongly connected components written by Gallagher; read is Warren and O'Keefe's public domain Prolog parser; ch_db_gr is a consistency checker for a lectures-administration database, written by Janssens; ch_db is the same program but using an open-ended list for the list of lectures to be checked; peephole is the optimizer of SB-Prolog, written by Debray; boyer is a Boyer-Moore theorem prover also from the Gabriel benchmarks (as translated by E. Tick); icomp is a code generator for the WAM, written by Demoen; ann is a simplified version of \&-Prolog's parallelizing annotator [21].

\section{Performance}

The following two tables correspond to Table 1 of Section 3.1 but show the analysis time and the time for stack-shifts and garbage collection separately. The meaning of the columns is as follows :

an: Analysis time.

ss+gc: Time for stack-shifts and garbage collection.

$\%$ : Percentage of the time for stack-shifts and garbage collection w.r.t. the total time for abstract interpretation of the benchmark programs.

\begin{tabular}{|c|c|c|c|c|c|c|c|c|c|c|c|c|}
\hline \multirow[b]{2}{*}{ Program } & \multicolumn{3}{|c|}{ alf-0 } & \multicolumn{3}{|c|}{ alf-1 } & \multicolumn{3}{|c|}{ alf-2 } & \multicolumn{3}{|c|}{ alf-3 } \\
\hline & an & $\mathrm{ss}+\mathrm{gc}$ & $\%$ & an & $\mathrm{ss}+\mathrm{gc}$ & $\%$ & an & $\mathbf{s s}+\mathbf{g c}$ & $\%$ & an & ss + gc & $\%$ \\
\hline serialize & 3.42 & 0.38 & 10.0 & 3.51 & 0.39 & 10.0 & 3.48 & 0.38 & 9.8 & 3.39 & 0.40 & 10.6 \\
\hline init_vars & 2.81 & 0.41 & 12.7 & 4.54 & 0.46 & 9.2 & 4.29 & 0.48 & 10.1 & 4.35 & 0.46 & 9.6 \\
\hline mapcolor' & 2.20 & 0.33 & 13.0 & 60.25 & 21.04 & 25.9 & 81.60 & 28.69 & 26.0 & 1119.74 & 419.46 & 27.3 \\
\hline mapcolor & 0.75 & 0.01 & 1.3 & 1.92 & 0.36 & 15.8 & 3.60 & 0.42 & 10.4 & 5.51 & 1.03 & 15.7 \\
\hline grammar & 0.44 & 0.02 & 4.3 & 0.60 & 0.01 & 1.6 & 0.55 & 0.02 & 3.5 & 0.56 & 0.01 & 1.8 \\
\hline browse & 11.18 & 1.03 & 8.4 & 23.45 & 4.65 & 16.5 & 9.74 & 1.69 & 14.8 & 14.85 & 2.41 & 14.0 \\
\hline browse_u & 25.93 & 3.17 & 10.9 & 38.80 & 6.75 & 14.8 & 10.09 & 1.69 & 14.3 & 14.96 & 2.41 & 13.9 \\
\hline bid & 2.28 & 0.41 & 15.2 & 3.43 & 0.45 & 11.6 & 5.00 & 1.02 & 16.9 & 6.75 & 1.08 & 13.8 \\
\hline rdtok & 18.59 & 3.52 & 15.9 & 19.94 & 3.67 & 15.5 & 20.06 & 3.62 & 15.3 & 20.16 & 3.65 & 15.3 \\
\hline deriv & 6.82 & 1.11 & 14.0 & 9.12 & 1.73 & 15.9 & 10.82 & 2.36 & 17.9 & 11.61 & 2.43 & 17.3 \\
\hline kalah & 9.03 & 1.74 & 16.2 & 15.92 & 3.04 & 16.0 & 24.77 & 4.82 & 16.3 & 50.18 & 9.59 & 16.0 \\
\hline $\operatorname{tarjan}$ & 25.56 & 4.30 & 14.4 & 51.93 & 9.08 & 14.9 & 106.16 & 18.37 & 14.8 & 994.69 & 176.43 & 15.1 \\
\hline read & 17.45 & 3.31 & 15.9 & 52.87 & 9.63 & 15.4 & 86.04 & 15.52 & 15.3 & 161.52 & 29.44 & 15.4 \\
\hline ch_db_gr & 16.00 & 3.27 & 17.0 & 23.07 & 4.58 & 16.6 & 37.43 & 7.57 & 16.8 & 52.86 & 10.17 & 16.1 \\
\hline ch_db & 18.73 & 3.86 & 17.1 & 26.53 & 5.17 & 16.3 & 42.26 & 8.25 & 16.3 & 58.06 & 10.83 & 15.7 \\
\hline peephole & 29.91 & 5.79 & 16.2 & 37.96 & 8.22 & 17.8 & 19.28 & 3.67 & 16.0 & 18.69 & 3.14 & 14.4 \\
\hline boyer & 18.50 & 2.58 & 12.2 & 47.83 & 6.87 & 12.6 & 101.26 & 13.13 & 11.5 & 197.48 & 23.95 & 10.8 \\
\hline icomp & 111.68 & 23.47 & 17.4 & 397.16 & 116.07 & 22.6 & 2762.84 & 987.26 & 26.3 & & & \\
\hline ann & 237.02 & 70.33 & 22.9 & & & & & & & & & \\
\hline
\end{tabular}




\begin{tabular}{|c|c|c|c|c|c|c|c|c|c|c|c|c|}
\hline \multirow[b]{2}{*}{ Program } & \multicolumn{3}{|c|}{ alf-0 } & \multicolumn{3}{|c|}{ al-0 } & \multicolumn{3}{|c|}{ af-0 } & \multicolumn{3}{|c|}{$a-0$} \\
\hline & an & $\mathrm{ss}+\mathrm{gc}$ & $\%$ & an & ss + gc & $\%$ & an & $\mathrm{ss}+\mathrm{gc}$ & $\%$ & an & $\mathrm{ss}+\mathrm{gc}$ & $\%$ \\
\hline serialize & 3.42 & 0.38 & 10.0 & 3.34 & 0.39 & 10.5 & 3.64 & 0.39 & 9.7 & 21.11 & 3.68 & 14.8 \\
\hline init_vars & 2.81 & 0.41 & 12.7 & 2.76 & 0.39 & 12.4 & 2.71 & 0.41 & 13.1 & 2.69 & 0.39 & 12.7 \\
\hline mapcolor' & 2.20 & 0.33 & 13.0 & 2.31 & 0.32 & 12.2 & 2.22 & 0.33 & 12.9 & 2.89 & 0.35 & 10.8 \\
\hline mapcolor & 0.75 & 0.01 & 1.3 & 0.83 & 0.02 & 2.4 & 0.79 & 0.01 & 1.2 & 1675.18 & 2.12 & 0.1 \\
\hline grammar & 0.44 & 0.02 & 4.3 & 0.44 & 0.02 & 4.3 & 0.44 & 0.00 & 0.0 & 0.42 & 0.00 & 0.0 \\
\hline browse & 11.18 & 1.03 & 8.4 & 11.11 & 1.00 & 8.3 & 12.03 & 1.59 & 11.7 & 638.31 & 1.85 & 0.3 \\
\hline browse_u & 25.93 & 3.17 & 10.9 & 26.51 & 2.64 & 9.1 & 26.95 & 3.16 & 10.5 & 252.01 & 4.17 & 1.6 \\
\hline bid & 2.28 & 0.41 & 15.2 & 2.32 & 0.42 & 15.3 & 2.27 & 0.42 & 15.6 & 2.30 & 0.43 & 15.8 \\
\hline rdtok & 18.59 & 3.52 & 15.9 & 13.72 & 2.39 & 14.8 & 15.90 & 3.01 & 15.9 & 16.77 & 3.01 & 15.2 \\
\hline deriv & 6.82 & 1.11 & 14.0 & 6.95 & 1.13 & 14.0 & 6.74 & 1.15 & 14.6 & 6.81 & 1.16 & 14.6 \\
\hline kalah & 9.03 & 1.74 & 16.2 & 8.96 & 1.77 & 16.5 & 8.92 & 1.80 & 16.8 & 8.77 & 1.75 & 16.6 \\
\hline tarjan & 25.56 & 4.30 & 14.4 & 26.22 & 4.47 & 14.6 & 25.27 & 4.25 & 14.4 & 36.19 & 7.24 & 16.7 \\
\hline read & 17.45 & $\mathbf{3 . 3 1}$ & 15.9 & 17.82 & 3.31 & 15.7 & 17.43 & 2.70 & 13.4 & 17.76 & 2.77 & 13.5 \\
\hline ch_db_gr & 16.00 & 3.27 & 17.0 & 16.19 & 3.26 & 16.8 & 15.89 & 2.71 & 14.6 & 15.96 & 2.73 & 14.6 \\
\hline ch_db & 18.73 & 3.86 & 17.1 & 18.94 & 3.88 & 17.0 & 67.03 & 21.85 & 24.6 & 363.20 & 115.67 & 24.2 \\
\hline peephole & 29.91 & 5.79 & 16.2 & 31.97 & 5.77 & 15.3 & 23.15 & 4.18 & 15.3 & 78.95 & 22.81 & 22.4 \\
\hline boyer & 18.50 & 2.58 & 12.2 & 19.05 & 2.59 & 12.0 & 85.28 & 26.85 & 23.9 & 86.64 & 26.72 & 23.6 \\
\hline icomp & 111.68 & 23.47 & 17.4 & 113.53 & 23.02 & 16.9 & 109.82 & 22.87 & 17.2 & 152.97 & 30.20 & 16.5 \\
\hline ann & 237.02 & 70.33 & 22.9 & 239.73 & 71.00 & 22.8 & 236.14 & 70.45 & 23.0 & 372.19 & 105.63 & 22.1 \\
\hline
\end{tabular}

The following table shows the various timing results for lattices $\{a, l, f\}$ and $\{a, l\}$ in the case of the $0^{*}$-analysis where the depth restriction is only imposed upon procedureentry. The table also shows the number of iterations (Its) for reaching a fix-point. The number of call patterns (Pat) analyzed in the 0 and $0^{*}$ analyses is identical (see Table 1 in Section 3.1); the analysis times are comparable except for the programs init_vars, mapcolor, and peephole for which the analysis $0^{*}$ is a factor 2 slower, the programs icomp and mapcolor' where $0^{*}$ is factors 3 and 6 slower respectively, and for the programs browse_u and ann where the analysis time for $0^{*}$ becomes unacceptable. The loss in performance can be explained by the presence of structural information and an increased average size for the sharing components (as for mapcolor', mapcolor, browse, browse_u, tarjan, ch_db, peephole, and icomp; see Table 3 in Section 3.1) and/or an increased number of iterations (which is the case for all programs except serialize and grammar; see tables of Appendix D).

\begin{tabular}{|c|c|c|c|c|c|c|c|c|c|c|c|c|c|c|}
\hline \multirow[b]{2}{*}{ Program } & \multicolumn{7}{|c|}{ alf- $0^{*}$} & \multicolumn{7}{|c|}{ al- $0^{*}$} \\
\hline & Its & Pat & sec & $\mathrm{Mb}$ & an & $s s+g c$ & $\%$ & Its & Pat & sec & $\mathrm{Mb}$ & an & ss $+g c$ & $\%$ \\
\hline serialize & 13 & 8 & 3.70 & 4.1 & 3.31 & 0.39 & 10.5 & 11 & 7 & 3.50 & 4.1 & 3.13 & 0.37 & 10.6 \\
\hline init_vars & 15 & 9 & 6.96 & 4.2 & 5.91 & 1.05 & 15.1 & 15 & 9 & 7.19 & 4.2 & 6.14 & 1.05 & 14.6 \\
\hline mapcolor' & 14 & 13 & 17.23 & 4.1 & 13.25 & 3.98 & 23.1 & 14 & 13 & 17.49 & 4.1 & 13.49 & 4.00 & 22.9 \\
\hline mapcolor & 8 & 8 & 1.57 & 4.1 & 1.53 & 0.04 & 2.5 & 8 & 8 & 1.62 & 4.1 & 1.58 & 0.04 & 2.5 \\
\hline grammar & 0 & 6 & 0.47 & 3.3 & 0.46 & 0.01 & 2.1 & 0 & 6 & 0.52 & 3.3 & 0.50 & 0.02 & 3.8 \\
\hline browse & 31 & 21 & 12.42 & 4.3 & 11.35 & 1.07 & 8.6 & 31 & 21 & 12.49 & 4.3 & 11.43 & 1.06 & 8.5 \\
\hline browse_u & 37 & 27 & 249.27 & 4.3 & 243.20 & 6.07 & 2.4 & 37 & 27 & 248.89 & 4.3 & 242.81 & 6.08 & 2.4 \\
\hline bid & 14 & 24 & 3.19 & 4.2 & 2.74 & 0.45 & 14.1 & 14 & 24 & 3.22 & 4.2 & 2.80 & 0.42 & 13.0 \\
\hline rdtok & 72 & 39 & 22.71 & 4.7 & 19.10 & 3.61 & 15.9 & 55 & 32 & 16.39 & 4.6 & 13.97 & 2.42 & 14.8 \\
\hline deriv & 27 & 31 & 9.20 & 4.3 & 7.51 & 1.69 & 18.4 & 27 & 31 & 9.40 & 4.3 & 7.70 & 1.70 & 18.1 \\
\hline kalah & 54 & 53 & 17.82 & 4.5 & 14.76 & 3.06 & 17.2 & 54 & 53 & 17.85 & 4.5 & 14.85 & 3.00 & 16.8 \\
\hline tarjan & 108 & 70 & 42.09 & 4.9 & 35.94 & 6.15 & 14.6 & 106 & 69 & 42.68 & 4.9 & 36.53 & 6.15 & 14.4 \\
\hline read & 28 & 27 & 21.20 & 4.7 & 17.86 & 3.34 & 15.8 & 28 & 27 & 21.48 & 4.7 & 18.19 & 3.29 & 15.3 \\
\hline ch_db_gr & 46 & 70 & 22.87 & 4.6 & 18.90 & 3.97 & 17.4 & 46 & 70 & 23.10 & 4.6 & 19.15 & 3.95 & 17.1 \\
\hline ch_db & 47 & 75 & 25.37 & 4.7 & 21.37 & 4.00 & 15.8 & 46 & 74 & 25.43 & 4.7 & 21.45 & 3.98 & 15.7 \\
\hline peephole & 75 & 42 & 66.62 & 4.7 & 55.42 & 11.20 & 16.8 & 81 & 44 & 69.05 & 4.7 & 57.82 & 11.23 & 16.3 \\
\hline boyer & 69 & 49 & 21.10 & 4.5 & 18.48 & 2.62 & 12.4 & 63 & 46 & 21.40 & 4.5 & 18.76 & 2.64 & 12.3 \\
\hline icomp & 185 & 165 & 440.31 & 5.8 & 343.02 & 97.29 & 22.1 & 178 & 154 & 441.49 & 5.7 & $\mathbf{3 4 4 . 3 0}$ & 97.19 & 22.0 \\
\hline
\end{tabular}




\section{Precision and Application of Optimization Rules}

First we show the table for depth 2 that corresponds to Table 4 of Section 3.1.

\begin{tabular}{|c|c|c|c|c|c|c|c|c|c|c|c|}
\hline Program & Ann-k & $\mathrm{Gr}$ & $\mathrm{Fr}$ & Lin & Any & Sin & NSin & Str & Sval & AvS & $\mathrm{MxS}$ \\
\hline \multirow[t]{4}{*}{ serialize } & alf- $2 \downarrow 0$ & 40 & 40 & 82 & 13 & 98 & 25 & 0 & 3 & 4.39 & 7 \\
\hline & al- $2 \downarrow 0$ & 40 & 0 & 122 & 13 & 98 & 25 & 0 & 3 & 4.39 & 7 \\
\hline & af $-2 \downarrow 0$ & 40 & 40 & 0 & 95 & 98 & 218 & 0 & 3 & 11.29 & 33 \\
\hline & $\mathrm{a}-2 \downarrow 0$ & 40 & 0 & 0 & 135 & 98 & 558 & 0 & 3 & 23.43 & 91 \\
\hline \multirow[t]{4}{*}{ init_vars } & alf- $2 \downarrow 0$ & 137 & 72 & 5 & 0 & 67 & 5 & 6 & 16 & 2.88 & $\begin{array}{lll}7 & & \end{array}$ \\
\hline & al-2 $\downarrow 0$ & 137 & 0 & 77 & 0 & 67 & 5 & 6 & 16 & 2.88 & 7 \\
\hline & af $-2 \downarrow 0$ & 137 & 72 & 0 & 5 & 67 & 5 & 6 & 16 & 2.88 & 7 \\
\hline & $\mathrm{a}-2 \downarrow 0$ & 137 & 0 & 0 & 77 & 67 & 5 & 6 & 16 & 2.88 & 7 \\
\hline \multirow[t]{4}{*}{ mapcolor' } & $\begin{array}{l}\text { alf- } 2 \downarrow 0 \\
\text { ald }\end{array}$ & 11 & 13 & 1 & 58 & 42 & 101 & 33 & 0 & 15.14 & 49 \\
\hline & al- $2 \downarrow 0$ & 11 & 0 & 14 & 58 & 42 & 101 & 33 & 0 & 15.14 & 49 \\
\hline & af $-2 \downarrow 0$ & 11 & 13 & 0 & 59 & 42 & 101 & 33 & 0 & 15.14 & 49 \\
\hline & $\mathrm{a}-2 \downarrow 0$ & 11 & 0 & 0 & 72 & 42 & 165 & 33 & 0 & 18.62 & 63 \\
\hline \multirow[t]{4}{*}{ mapcolor } & alf- $2 \downarrow 0$ & 52 & 25 & 6 & 10 & 33 & 10 & 42 & 0 & 3.77 & 10 \\
\hline & al- $2 \downarrow 0$ & 52 & 0 & 31 & 10 & 33 & 10 & 42 & 0 & 3.77 & 10 \\
\hline & af $-2 \downarrow 0$ & 52 & 25 & 0 & 16 & 33 & 13 & 42 & 0 & 4.00 & 10 \\
\hline & $\mathrm{a}-2 \downarrow 0$ & 52 & 0 & 0 & 41 & 33 & 1083 & 42 & 0 & 87.00 & 1023 \\
\hline \multirow[t]{4}{*}{ grammar } & alf- $2 \downarrow 0$ & 37 & 13 & 2 & 0 & 15 & 0 & 17 & 4 & 1.88 & 5 \\
\hline & al- $2 \downarrow 0$ & 37 & 0 & 15 & 0 & 15 & 0 & 17 & 4 & 1.88 & 5 \\
\hline & af- $2 \downarrow 0$ & 37 & 13 & 0 & 2 & 15 & 0 & 17 & 4 & 1.88 & 5 \\
\hline & $\mathrm{a}-2 \downarrow 0$ & 37 & 0 & 0 & 15 & 15 & 0 & 17 & 4 & 1.88 & 5 \\
\hline \multirow[t]{4}{*}{ browse } & alf- $2 \downarrow 0$ & 214 & 172 & 109 & 73 & 279 & 72 & 124 & 12 & 4.29 & 14 \\
\hline & al- $2 \downarrow 0$ & 214 & 0 & 281 & 73 & 279 & 72 & 124 & 12 & 4.29 & 14 \\
\hline & af $-2 \downarrow 0$ & 214 & 172 & 0 & 182 & 279 & 229 & 124 & 12 & 6.46 & 71 \\
\hline & $\mathrm{a}-2 \downarrow 0$ & 214 & 0 & 0 & 354 & 279 & 1217 & 124 & 12 & 17.59 & 512 \\
\hline \multirow[t]{4}{*}{ browse_u } & alf- $2 \downarrow 0$ & 176 & 125 & 103 & 70 & 245 & 53 & 99 & 7 & 3.94 & 14 \\
\hline & al- $2 \downarrow 0$ & 176 & 0 & 228 & 70 & 245 & 53 & 99 & 7 & 3.94 & 14 \\
\hline & af $-2 \downarrow 0$ & 176 & 125 & 0 & 173 & 245 & 209 & 99 & 7 & 6.24 & 71 \\
\hline & $\mathrm{a}-2 \downarrow 0$ & 176 & 0 & 0 & 298 & 245 & 693 & 99 & 7 & 12.26 & 512 \\
\hline \multirow[t]{4}{*}{ bid } & alf- $2 \downarrow 0$ & 340 & 123 & 1 & 0 & 124 & 0 & 91 & 0 & 2.25 & 8 \\
\hline & al- $2 \downarrow 0$ & 340 & 0 & 124 & 0 & 124 & 0 & 91 & 0 & 2.25 & 8 \\
\hline & af- $2 \downarrow 0$ & 340 & 123 & 0 & 1 & 124 & 0 & 91 & 0 & 2.25 & 8 \\
\hline & $\mathrm{a}-2 \downarrow 0$ & 340 & 0 & 0 & 124 & 124 & 34 & 91 & 0 & 2.87 & 19 \\
\hline \multirow[t]{4}{*}{ rdtok } & alf- $2 \downarrow 0$ & 337 & 320 & 147 & 41 & 496 & 42 & 8 & 5 & 2.85 & 6 \\
\hline & al- $2 \downarrow 0$ & 337 & 0 & 467 & 41 & 496 & 42 & 8 & 5 & 2.85 & 6 \\
\hline & af $-2 \downarrow 0$ & 337 & 320 & 0 & 188 & 496 & 48 & 8 & 5 & 2.93 & 13 \\
\hline & $\mathrm{a}-2 \downarrow 0$ & 337 & 0 & 0 & 508 & 496 & 269 & 8 & 5 & 4.09 & 17 \\
\hline \multirow[t]{4}{*}{ deriv } & alf- $2 \downarrow 0$ & 523 & 193 & $\overline{0}$ & 0 & 193 & 0 & 26 & 7 & 1.74 & 4 \\
\hline & al- $2 \downarrow 0$ & 523 & 0 & 193 & 0 & 193 & 0 & 26 & 7 & 1.74 & 4 \\
\hline & af- $2 \downarrow 0$ & 523 & 193 & 0 & 0 & 193 & 0 & 26 & 7 & 1.74 & 4 \\
\hline & $\mathrm{a}-2 \downarrow 0$ & 523 & 0 & 0 & 193 & 193 & 0 & 26 & 7 & 1.74 & 4 \\
\hline \multirow[t]{4}{*}{ kalah } & alf- $2 \downarrow 0$ & 958 & 237 & 28 & 5 & 270 & 1 & 511 & 12 & 2.04 & 7 \\
\hline & al- $2 \downarrow 0$ & 958 & 0 & 265 & 5 & 270 & 1 & 511 & 12 & 2.04 & 7 \\
\hline & af- $2 \downarrow 0$ & 958 & 237 & 0 & 33 & 270 & 2 & 511 & 12 & 2.05 & 7 \\
\hline & $\mathrm{a}-2 \downarrow 0$ & 958 & 0 & 0 & 270 & 270 & 10 & 511 & 12 & 2.18 & 7 \\
\hline \multirow[t]{4}{*}{ tarjan } & alf- $2 \downarrow 0$ & 1037 & 449 & 122 & 299 & 817 & 343 & 489 & 211 & 4.62 & 35 \\
\hline & al- $2 \downarrow 0$ & 1037 & 0 & 571 & 299 & 817 & 343 & 489 & 211 & 4.62 & 35 \\
\hline & af- $2 \downarrow 0$ & 1037 & 449 & 0 & 421 & 817 & 389 & 489 & 211 & 4.88 & 35 \\
\hline & $\mathrm{a}-2 \downarrow 0$ & 1037 & 0 & 0 & 870 & 817 & 680 & 489 & 211 & 5.96 & 69 \\
\hline \multirow[t]{4}{*}{ read } & alf- $2 \downarrow 0$ & 1370 & 709 & 4 & 4 & 715 & 2 & 164 & 57 & 3.32 & 8 \\
\hline & al-2 $\downarrow 0$ & 1370 & 0 & 713 & 4 & 715 & 2 & 164 & 57 & 3.32 & 8 \\
\hline & af $-2 \downarrow 0$ & 1370 & 709 & 0 & 8 & 715 & 2 & 164 & 57 & 3.32 & 8 \\
\hline & $\mathrm{a}-2 \downarrow 0$ & 1370 & 0 & 0 & 717 & 715 & 17 & 164 & 57 & 3.39 & 8 \\
\hline
\end{tabular}




\begin{tabular}{|c|c|c|c|c|c|c|c|c|c|c|c|}
\hline Program & Ann-k & Gr & Fr & Lin & Any & Sin & NSin & Str & Sval & $\mathrm{AvS}$ & $\mathrm{MxS}$ \\
\hline \multirow[t]{4}{*}{ ch_db_gr } & alf- $2 \downarrow 0$ & 1134 & 216 & 1 & 16 & 231 & 8 & 210 & 18 & 2.49 & 8 \\
\hline & al- $2 \downarrow 0$ & 1134 & 0 & 217 & 16 & 231 & 8 & 210 & 18 & 2.49 & 8 \\
\hline & af- $2 \downarrow 0$ & 1134 & 216 & 0 & 17 & 231 & 8 & 210 & 18 & 2.49 & 8 \\
\hline & $\mathrm{a}-2 \downarrow 0$ & 1134 & 0 & 0 & 233 & 231 & 40 & 210 & 18 & 2.85 & 9 \\
\hline \multirow[t]{4}{*}{ ch_db } & alf- $2 \downarrow 0$ & 910 & 221 & 214 & 31 & 433 & 34 & 211 & 18 & 2.88 & 11 \\
\hline & al- $2 \downarrow 0$ & 910 & 0 & 435 & 31 & 433 & 34 & 211 & 18 & 2.88 & 11 \\
\hline & af $-2 \downarrow 0$ & 910 & 221 & 0 & 245 & 433 & 678 & 211 & 18 & 6.81 & 63 \\
\hline & $\mathrm{a}-2 \downarrow 0$ & 910 & 0 & 0 & 466 & 433 & 1002 & 211 & 18 & 8.63 & 127 \\
\hline \multirow[t]{4}{*}{ peephole } & alf- $2 \downarrow 0$ & 593 & 211 & 281 & 15 & 358 & 101 & 96 & 32 & 2.30 & 9 \\
\hline & al- $2 \downarrow 0$ & 593 & 0 & 492 & 15 & 358 & 101 & 96 & 32 & 2.30 & 9 \\
\hline & af- $2 \downarrow 0$ & 593 & 211 & 0 & 296 & 358 & 396 & 96 & 32 & 4.02 & 33 \\
\hline & $\mathrm{a}-2 \downarrow 0$ & 593 & 0 & 0 & 507 & 358 & 702 & 96 & 32 & 5.61 & 64 \\
\hline \multirow[t]{2}{*}{ boyer } & alf- $2 \downarrow 0$ & 137 & 66 & 447 & 48 & 466 & 120 & 74 & 28 & 2.85 & 18 \\
\hline & al- $2 \downarrow 0$ & 137 & 0 & 513 & 48 & 466 & 120 & 74 & 28 & 2.85 & 18 \\
\hline \multirow[t]{4}{*}{ icomp } & alf- $2 \downarrow 0$ & 816 & 1032 & 131 & 1419 & 1927 & 2504 & 155 & 181 & 8.81 & 208 \\
\hline & al- $2 \downarrow 0$ & 816 & 0 & 1163 & 1419 & 1927 & 2504 & 155 & 181 & 8.81 & 208 \\
\hline & af $-2 \downarrow 0$ & 816 & 1032 & 0 & 1550 & 1927 & 2504 & 155 & 181 & 8.81 & 208 \\
\hline & a- $2 \downarrow 0$ & 816 & 0 & 0 & 2582 & 1927 & 3676 & 155 & 181 & 10.98 & 208 \\
\hline
\end{tabular}

Next follow the full tables concerning the application of optimization rules during unification, which correspond to the summary Table 2 of Section 3.1. The meaning of the columns is as follows :

Its: Number of iterations.

Pat: Number of call-success patterns analyzed.

FR: Percentage of applications of freeness rule $(*)$.

L1R: Percentage of applications of linear rule (one-side) $\left(^{* *}\right)$.

L2R: Percentage of applications of linear rule (both-sides) $\left(^{* *}\right)$.

GrR: Percentage of applications of groundness rule (o).

NoR: Percentage of applications of general rule.

Unif: Total number of calls for unification operations (Algorithm 3). 


\begin{tabular}{|c|c|c|c|c|c|c|c|c|c|}
\hline Program & Ann-k & $\overline{\text { Its }}$ & Pat & FR & L1R & L2R & GrR & NoR & $\overline{\text { Unif }}$ \\
\hline serialize & $\overline{\text { alf-0 }}$ & 13 & 8 & 39.5 & 4.1 & 14.9 & 41.6 & 0.0 & 296 \\
\hline serialize & alf-1 & 13 & 8 & 39.7 & 4.0 & 14.5 & 41.8 & 0.0 & 297 \\
\hline serialize & alf-2 & 13 & 8 & 39.7 & 4.0 & 14.5 & 41.8 & 0.0 & 297 \\
\hline init_vars & alf-0 & 7 & 9 & 19.4 & 0.0 & 0.0 & 80.6 & 0.0 & 299 \\
\hline init_vars & alf-1 & 11 & 9 & 17.2 & 0.0 & 0.0 & 82.8 & 0.0 & 442 \\
\hline init_vars & alf-2 & 11 & 9 & 17.2 & 0.0 & 0.0 & 82.8 & 0.0 & 442 \\
\hline mapcolor' & alf-0 & $\overline{11}$ & 13 & 51.1 & 7.0 & 2.7 & 19.4 & 19.9 & 186 \\
\hline mapcolor' & alf-1 & 25 & 22 & 54.0 & 4.9 & 9.0 & 11.8 & 20.3 & 365 \\
\hline mapcolor' & alf-2 & 28 & 24 & 54.9 & 5.3 & 8.8 & 10.3 & 20.8 & 399 \\
\hline mapcolor & alf-0 & 4 & 8 & $\overline{18.7}$ & 1.9 & 0.9 & 78.5 & 0.0 & $\overline{107}$ \\
\hline mapcolor & alf-1 & 14 & 19 & 15.4 & 1.1 & 1.1 & 82.3 & 0.0 & 266 \\
\hline mapcolor & alf-2 & 27 & 27 & 13.7 & 0.5 & 0.5 & 85.4 & 0.0 & 437 \\
\hline grammar & alf-0 & 0 & 6 & 16.3 & 0.0 & 5.1 & 78.6 & 0.0 & 98 \\
\hline grammar & alf-1 & 0 & 6 & 9.0 & 0.0 & 0.0 & 91.0 & 0.0 & 111 \\
\hline grammar & alf-2 & 0 & 6 & 9.0 & 0.0 & 0.0 & 91.0 & 0.0 & 111 \\
\hline browse & alf-0 & 25 & 21 & 26.2 & 3.8 & 6.8 & 58.6 & 4.6 & $\overline{497}$ \\
\hline browse & alf-1 & 31 & 24 & 25.3 & 3.3 & 6.6 & 58.9 & 5.9 & 574 \\
\hline browse & alf-2 & 46 & 37 & 28.6 & 3.6 & 13.8 & 46.9 & 7.0 & 908 \\
\hline browse_u & alf-0 & 31 & 27 & 27.3 & 3.2 & 8.0 & 57.0 & 4.4 & 589 \\
\hline browse_u & alf-1 & 37 & 30 & 26.3 & 2.8 & 7.6 & 57.2 & 6.0 & 669 \\
\hline browse_u & alf-2 & 51 & 42 & 28.9 & 3.3 & 13.8 & 47.3 & 6.7 & 987 \\
\hline bid & alf-0 & 7 & 24 & 4.8 & 0.0 & 0.0 & 95.2 & 0.0 & 377 \\
\hline bid & alf-1 & 15 & 29 & 4.8 & 0.0 & 0.0 & 95.2 & 0.0 & 497 \\
\hline bid & alf-2 & 22 & 34 & 4.7 & 0.0 & 0.0 & 95.3 & 0.0 & 639 \\
\hline rdtok & alf-0 & 59 & 39 & 41.4 & 0.2 & 6.4 & 51.7 & 0.3 & 2018 \\
\hline rdtok & alf-1 & 71 & 39 & 40.4 & 0.1 & 5.8 & 53.5 & 0.1 & 2130 \\
\hline rdtok & alf-2 & 73 & 40 & 40.0 & 0.1 & 5.7 & 54.0 & 0.1 & 2150 \\
\hline deriv & alf-0 & 22 & 31 & 7.1 & 0.0 & 0.0 & 92.9 & 0.0 & 926 \\
\hline deriv & alf-1 & 30 & 40 & 6.4 & 0.0 & 0.0 & 93.6 & 0.0 & 1172 \\
\hline deriv & alf-2 & 37 & 46 & 6.4 & 0.0 & 0.0 & 93.6 & 0.0 & 1344 \\
\hline kalah & alf-0 & 32 & 53 & 7.1 & 0.1 & 0.1 & 92.7 & 0.0 & 1002 \\
\hline kalah & alf-1 & 37 & 54 & 5.9 & 0.1 & 0.1 & 94.0 & 0.0 & 1356 \\
\hline kalah & alf-2 & 54 & 61 & 5.1 & 0.1 & 0.1 & 94.8 & 0.0 & 1810 \\
\hline $\operatorname{tarjan}$ & alf-0 & 85 & 70 & 26.4 & 2.8 & 2.7 & 62.2 & 6.0 & 2246 \\
\hline tarjan & alf-1 & 124 & 96 & 26.6 & 1.6 & 5.9 & 62.1 & 3.9 & 3998 \\
\hline tarjan & alf-2 & 188 & 126 & 20.9 & 1.0 & 4.2 & 71.1 & 2.8 & 6569 \\
\hline read & alf-0 & 22 & 27 & 10.8 & 0.0 & 0.0 & 89.2 & 0.0 & 1560 \\
\hline read & alf-1 & 52 & 43 & 7.4 & 0.0 & 0.0 & 92.6 & 0.0 & 3869 \\
\hline read & alf-2 & 86 & 58 & 6.3 & 0.0 & 0.0 & 93.7 & 0.0 & 5767 \\
\hline ch_db_gr & alf-0 & 28 & 70 & 4.3 & 0.1 & 0.3 & 95.0 & 0.2 & 2072 \\
\hline ch_db_gr & alf-1 & 41 & 82 & 3.6 & 0.0 & 0.0 & 96.2 & 0.2 & 2604 \\
\hline ch_db_gr & alf-2 & 60 & 93 & 3.3 & 0.0 & 0.0 & 96.6 & 0.1 & 3543 \\
\hline ch_db & alf-0 & 34 & 75 & 7.9 & 0.5 & 2.8 & 88.5 & 0.2 & 2441 \\
\hline$c h \_d b$ & alf-1 & 48 & 89 & 8.7 & 0.4 & 3.2 & 87.5 & 0.2 & 3010 \\
\hline ch_db & alf-2 & 69 & 102 & 7.8 & 0.3 & 2.3 & 89.5 & 0.1 & 4022 \\
\hline peephole & alf-0 & 63 & 42 & 34.8 & 4.2 & 2.6 & 50.7 & 7.7 & 2361 \\
\hline peephole & alf-1 & 67 & 42 & 34.2 & 3.2 & 2.5 & 52.7 & 7.3 & 2493 \\
\hline peephole & alf-2 & 44 & 36 & 32.7 & 0.6 & 6.3 & 60.4 & 0.0 & 2135 \\
\hline boyer & alf-0 & 64 & 49 & 51.9 & 4.1 & 20.8 & 22.5 & 0.7 & 1922 \\
\hline boyer & alf-1 & 120 & 69 & 47.8 & 5.0 & 26.5 & 20.3 & 0.4 & 3457 \\
\hline boyer & alf-2 & 192 & 94 & 44.3 & 5.3 & 32.5 & 17.7 & 0.2 & 5749 \\
\hline icomp & alf-0 & 179 & 165 & 49.0 & 4.3 & 0.8 & 32.5 & 13.4 & 5858 \\
\hline icomp & alf-1 & 220 & 204 & 47.5 & 3.7 & 0.6 & 35.0 & 13.1 & 7180 \\
\hline icomp & alf-2 & 259 & 239 & 44.9 & 3.5 & 0.5 & 37.5 & 13.6 & 8355 \\
\hline
\end{tabular}




\begin{tabular}{|c|c|c|c|c|c|c|c|c|c|}
\hline Program & Ann-k & Its & Pat & $\overline{\mathrm{FR}}$ & L1R & L2R & GrR & NoR & Unif \\
\hline serialize & $\overline{\text { al-0 }}$ & 11 & 7 & $\overline{0.0}$ & 8.9 & 52.8 & $\overline{38.3}$ & 0.0 & $\overline{269}$ \\
\hline serialize & al-1 & 11 & 7 & 0.0 & 8.9 & 52.6 & 38.5 & 0.0 & 270 \\
\hline serialize & al-2 & 11 & 7 & 0.0 & 8.9 & 52.6 & 38.5 & 0.0 & 270 \\
\hline init_vars & al-0 & 6 & 9 & 0.0 & 0.0 & 18.4 & 81.6 & 0.0 & 293 \\
\hline init_vars & al-1 & 11 & 9 & 0.0 & 0.0 & 17.2 & 82.8 & 0.0 & 442 \\
\hline init_vars & al-2 & 11 & 9 & 0.0 & 0.0 & 17.2 & 82.8 & 0.0 & 442 \\
\hline mapcolor' & al-0 & 10 & 13 & 0.0 & 40.6 & 18.9 & 20.0 & 20.6 & 180 \\
\hline mapcolor' & al-1 & 25 & 22 & 0.0 & 36.2 & 31.8 & 11.8 & 20.3 & 365 \\
\hline mapcolor' & al-2 & 26 & 23 & 0.0 & 36.4 & 31.4 & 10.6 & 21.6 & 385 \\
\hline mapcolor & al-0 & 4 & 8 & 0.0 & 9.3 & $\overline{12.1}$ & 78.5 & 0.0 & 107 \\
\hline mapcolor & al-1 & 12 & 17 & 0.0 & 6.0 & 11.5 & 82.5 & 0.0 & 234 \\
\hline mapcolor & al-2 & 20 & 22 & 0.0 & 3.9 & 10.4 & 85.6 & 0.0 & 355 \\
\hline grammar & al-0 & 0 & 6 & 0.0 & 0.0 & $\overline{21.4}$ & 78.6 & 0.0 & 98 \\
\hline grammar & al-1 & 0 & 6 & 0.0 & 0.0 & 9.0 & 91.0 & 0.0 & 111 \\
\hline grammar & al-2 & 0 & 6 & 0.0 & 0.0 & 9.0 & 91.0 & 0.0 & 111 \\
\hline browse & $\overline{a l-0}$ & 24 & 21 & 0.0 & 11.9 & 26.5 & 56.7 & 4.9 & 471 \\
\hline browse & al-1 & 30 & 24 & 0.0 & 10.8 & 25.9 & 57.1 & 6.2 & 545 \\
\hline browse & al-2 & 45 & 37 & 0.0 & 12.7 & 34.9 & 45.1 & 7.3 & 875 \\
\hline browse_u & $\overline{a l-0}$ & 30 & 27 & 0.0 & $\overline{11.7}$ & 28.1 & 55.6 & 4.6 & 565 \\
\hline browse_u & al-1 & 36 & 30 & 0.0 & 10.7 & 27.2 & 55.8 & 6.2 & 643 \\
\hline browse_u & al-2 & 50 & 42 & 0.0 & 11.6 & 35.6 & 45.9 & 6.9 & 957 \\
\hline bid & al-0 & 7 & 24 & 0.0 & 0.0 & 4.8 & 95.2 & 0.0 & 377 \\
\hline bid & al-1 & 15 & 29 & 0.0 & 0.0 & 4.8 & 95.2 & 0.0 & 497 \\
\hline bid & al-2 & 22 & 34 & 0.0 & 0.0 & 4.7 & 95.3 & 0.0 & 639 \\
\hline rdtok & al-0 & 44 & 32 & 0.0 & 10.4 & 33.3 & 55.9 & 0.4 & 1434 \\
\hline rdtok & al-1 & 54 & 32 & 0.0 & 9.6 & 32.1 & 58.2 & 0.2 & 1525 \\
\hline rdtok & al-2 & 56 & 33 & 0.0 & 9.4 & 31.7 & 58.7 & 0.2 & 1545 \\
\hline deriv & al-0 & 22 & 31 & 0.0 & 0.0 & 7.1 & 92.9 & 0.0 & 926 \\
\hline deriv & al-1 & 30 & 40 & 0.0 & 0.0 & 6.4 & 93.6 & 0.0 & 1172 \\
\hline deriv & al-2 & 37 & 46 & 0.0 & 0.0 & 6.4 & 93.6 & 0.0 & 1344 \\
\hline kalah & al-0 & 31 & 53 & 0.0 & 0.3 & 7.1 & 92.6 & 0.0 & 984 \\
\hline kalah & al-1 & 36 & 54 & 0.0 & 0.2 & 5.9 & 93.8 & 0.0 & 1331 \\
\hline kalah & al-2 & 53 & 61 & 0.0 & 0.2 & 5.1 & 94.7 & 0.0 & 1781 \\
\hline tarjan & al-0 & 84 & 69 & 0.0 & 14.4 & 17.6 & 61.9 & 6.1 & 2231 \\
\hline $\operatorname{tarjan}$ & al-1 & 118 & 96 & 0.0 & 9.2 & 24.4 & 62.6 & 3.8 & 3844 \\
\hline tarjan & al-2 & 180 & 126 & 0.0 & 6.4 & 19.2 & 71.7 & 2.7 & 6347 \\
\hline read & al-0 & 22 & 27 & 0.0 & 0.1 & 10.6 & 89.2 & 0.0 & 1560 \\
\hline read & al-1 & 52 & 43 & 0.0 & 0.1 & 7.4 & 92.6 & 0.0 & 3869 \\
\hline read & al-2 & 86 & 58 & 0.0 & 0.0 & 6.3 & 93.7 & 0.0 & 5767 \\
\hline ch_db_gr & $\overline{\text { al-0 }}$ & 28 & 70 & 0.0 & 0.7 & $\overline{4.1}$ & 95.0 & 0.2 & 2072 \\
\hline ch_db_gr & al-1 & 41 & 82 & 0.0 & 0.4 & 3.2 & 96.2 & 0.2 & 2604 \\
\hline ch_db_gr & al-2 & 60 & 93 & 0.0 & 0.3 & 3.0 & 96.6 & 0.1 & 3543 \\
\hline ch_db & $\overline{a l-0}$ & 33 & 74 & 0.0 & 1.4 & 9.6 & 88.8 & 0.2 & 2431 \\
\hline ch_db & al-1 & 48 & 89 & 0.0 & 1.1 & 11.3 & 87.5 & 0.2 & 3010 \\
\hline ch_db & al-2 & 69 & 102 & 0.0 & 0.8 & 9.6 & 89.5 & 0.1 & 4022 \\
\hline peephole & al-0 & 67 & 44 & 0.0 & 19.8 & 22.5 & 49.9 & 7.8 & 2427 \\
\hline peephole & al-1 & 72 & 44 & 0.0 & 18.6 & 22.0 & 51.9 & 7.4 & 2574 \\
\hline peephole & al-2 & 37 & 30 & 0.0 & 1.6 & 34.3 & 64.1 & 0.0 & 1966 \\
\hline boyer & al-0 & 59 & 46 & 0.0 & 13.4 & 63.3 & 22.9 & 0.4 & 1874 \\
\hline boyer & al-1 & 115 & 67 & 0.0 & 12.5 & 66.8 & 20.4 & 0.2 & 3409 \\
\hline boyer & al-2 & 187 & 92 & 0.0 & 11.8 & 70.3 & 17.8 & 0.1 & 5701 \\
\hline icomp & al-0 & 172 & 154 & 0.0 & 36.5 & 17.3 & 32.5 & 13.7 & 5567 \\
\hline icomp & al-1 & 213 & 193 & 0.0 & 34.8 & 16.7 & 35.1 & 13.3 & 6889 \\
\hline icomp & al-2 & 252 & 228 & 0.0 & 33.7 & 14.9 & 37.6 & 13.8 & 8064 \\
\hline
\end{tabular}




\begin{tabular}{|c|c|c|c|c|c|c|c|c|c|}
\hline Program & Ann-k & Its & Pat & $\overline{F R}$ & L1R & L2R & GrR & NoR & $\overline{\text { Unif }}$ \\
\hline serialize & $\overline{\text { af-0 }}$ & 12 & 8 & 37.4 & 0.0 & 0.0 & 44.2 & 18.5 & 265 \\
\hline serialize & af-1 & 12 & 8 & 37.6 & 0.0 & 0.0 & 44.4 & 18.0 & 266 \\
\hline serialize & af-2 & 12 & 8 & 37.6 & 0.0 & 0.0 & 44.4 & 18.0 & 266 \\
\hline init_vars & af-0 & 7 & 9 & 19.4 & 0.0 & 0.0 & 80.6 & 0.0 & 299 \\
\hline init_vars & af-1 & 11 & 9 & 17.2 & 0.0 & 0.0 & 82.8 & 0.0 & 442 \\
\hline init_vars & af-2 & 11 & 9 & 17.2 & 0.0 & 0.0 & 82.8 & 0.0 & 442 \\
\hline mapcolor' & $\overline{\text { af-0 }}$ & 11 & 13 & 51.1 & 0.0 & 0.0 & 19.4 & 29.6 & 186 \\
\hline mapcolor' & af-1 & 18 & 17 & 52.4 & 0.0 & 0.0 & 14.7 & 33.0 & 273 \\
\hline mapcolor' & af-2 & 24 & 23 & 55.2 & 0.0 & 0.0 & 11.0 & 33.7 & 362 \\
\hline mapcolor & $\overline{\text { af-0 }}$ & 4 & 8 & $\overline{18.7}$ & 0.0 & 0.0 & 78.5 & 2.8 & $\overline{107}$ \\
\hline mapcolor & af-1 & 10 & 15 & 17.1 & 0.0 & 0.0 & 81.1 & 1.8 & 217 \\
\hline mapcolor & af-2 & 23 & 24 & 14.1 & 0.0 & 0.0 & 85.1 & 0.8 & 397 \\
\hline grammar & af-0 & 0 & 6 & 16.3 & 0.0 & 0.0 & 78.6 & 5.1 & 98 \\
\hline grammar & af-1 & 0 & 6 & 9.0 & 0.0 & 0.0 & 91.0 & 0.0 & 111 \\
\hline grammar & af-2 & 0 & 6 & 9.0 & 0.0 & 0.0 & 91.0 & 0.0 & 111 \\
\hline browse & $\overline{a f-0}$ & 31 & 24 & 28.2 & 0.0 & 0.0 & 53.4 & 18.4 & 581 \\
\hline browse & af-1 & 38 & 27 & 27.0 & 0.0 & 0.0 & 54.5 & 18.4 & 662 \\
\hline browse & af-2 & 41 & 31 & 26.5 & 0.0 & 0.0 & 51.7 & 21.9 & 805 \\
\hline browse_u & $\overline{a f-0}$ & 37 & 30 & 29.0 & 0.0 & 0.0 & 52.7 & 18.3 & 673 \\
\hline browse_u & af-1 & 44 & 33 & 27.7 & 0.0 & 0.0 & 53.6 & 18.6 & 757 \\
\hline browse_u & af-2 & 47 & 37 & 26.9 & 0.0 & 0.0 & 50.9 & 22.2 & 906 \\
\hline bid & $\overline{a f-0}$ & 7 & 24 & 4.8 & 0.0 & 0.0 & 95.2 & 0.0 & 377 \\
\hline bid & af-1 & 15 & 29 & 4.8 & 0.0 & 0.0 & 95.2 & 0.0 & 497 \\
\hline bid & af-2 & 22 & 34 & 4.7 & 0.0 & 0.0 & 95.3 & 0.0 & 639 \\
\hline rdtok & af-0 & 52 & 39 & 41.1 & 0.0 & 0.0 & 52.8 & 6.1 & 1785 \\
\hline rdtok & af-1 & 63 & 39 & 40.1 & 0.0 & 0.0 & 54.8 & 5.1 & 1891 \\
\hline rdtok & af-2 & 65 & 40 & 39.7 & 0.0 & 0.0 & 55.3 & 5.0 & 1911 \\
\hline deriv & af-0 & 22 & 31 & 7.1 & 0.0 & 0.0 & 92.9 & 0.0 & 926 \\
\hline deriv & af-1 & 30 & 40 & 6.4 & 0.0 & 0.0 & 93.6 & 0.0 & 1172 \\
\hline deriv & af-2 & 37 & 46 & 6.4 & 0.0 & 0.0 & 93.6 & 0.0 & 1344 \\
\hline kalah & af-0 & 32 & 53 & 7.1 & 0.0 & 0.0 & 92.7 & 0.2 & 1002 \\
\hline kalah & af-1 & 37 & 54 & 5.9 & 0.0 & 0.0 & 94.0 & 0.1 & 1356 \\
\hline kalah & af- 2 & 54 & 61 & 5.1 & 0.0 & 0.0 & 94.8 & 0.1 & 1810 \\
\hline $\operatorname{tarjan}$ & af-0 & 85 & 70 & 26.4 & 0.0 & 0.0 & 62.2 & 11.5 & 2246 \\
\hline tarjan & af-1 & 101 & 80 & 24.3 & 0.0 & 0.0 & 65.3 & 10.4 & 3085 \\
\hline tarjan & af-2 & 154 & 106 & 18.5 & 0.0 & 0.0 & 74.7 & 6.8 & 5180 \\
\hline read & af-0 & 22 & 27 & 10.8 & 0.0 & 0.0 & 89.2 & 0.0 & 1560 \\
\hline read & af-1 & 52 & 43 & 7.4 & 0.0 & 0.0 & 92.6 & 0.0 & 3869 \\
\hline read & af-2 & 86 & 58 & 6.3 & 0.0 & 0.0 & 93.7 & 0.0 & 5767 \\
\hline ch_db_gr & af-0 & 28 & 70 & 4.3 & 0.0 & 0.0 & 95.0 & 0.7 & 2072 \\
\hline ch_db_gr & af-1 & 41 & 82 & 3.6 & 0.0 & 0.0 & 96.2 & 0.2 & 2604 \\
\hline ch_db_gr & af-2 & 60 & 93 & 3.3 & 0.0 & 0.0 & 96.6 & 0.1 & 3543 \\
\hline ch_db & af-0 & 33 & 75 & 7.8 & 0.0 & 0.0 & 88.7 & 3.5 & 2437 \\
\hline$c h \_d b$ & af-1 & 47 & 89 & 8.7 & 0.0 & 0.0 & 87.6 & 3.7 & 3006 \\
\hline ch_db & af-2 & 68 & 102 & 7.7 & 0.0 & 0.0 & 89.6 & 2.6 & 4018 \\
\hline peephole & af-0 & $\overline{51}$ & 39 & 34.4 & 0.0 & 0.0 & 54.6 & 11.0 & 2155 \\
\hline peephole & af-1 & 60 & 40 & 34.0 & 0.0 & 0.0 & 54.5 & 11.5 & 2402 \\
\hline peephole & af-2 & 44 & 36 & 32.7 & 0.0 & 0.0 & 60.4 & 6.9 & 2135 \\
\hline boyer & af-0 & 64 & 49 & 51.9 & 0.0 & 0.0 & 22.5 & 25.6 & 1922 \\
\hline icomp & af-0 & 177 & 165 & 49.0 & 0.0 & 0.0 & 32.5 & 18.4 & 5838 \\
\hline icomp & af-1 & 218 & 204 & 47.5 & 0.0 & 0.0 & 35.1 & 17.4 & 7160 \\
\hline icomp & af-2 & 257 & 239 & 44.9 & 0.0 & 0.0 & 37.5 & 17.6 & 8335 \\
\hline
\end{tabular}




\begin{tabular}{|c|c|c|c|c|c|c|c|c|c|}
\hline Program & Ann-k & Its & Pat & $\overline{\mathrm{FR}}$ & $\overline{\mathrm{L} 1 \mathrm{R}}$ & L2R & GrR & NoR & Unif \\
\hline serialize & $a-0$ & 20 & 13 & 0.0 & 0.0 & 0.0 & 36.1 & 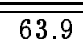 & 551 \\
\hline serialize & a-1 & 20 & 13 & 0.0 & 0.0 & 0.0 & 36.6 & 63.4 & 555 \\
\hline serialize & a-2 & 20 & 13 & 0.0 & 0.0 & 0.0 & 36.6 & 63.4 & 555 \\
\hline init_vars & $a-0$ & 6 & 9 & 0.0 & 0.0 & 0.0 & 81.6 & 18.4 & 293 \\
\hline init_vars & a-1 & 11 & 9 & 0.0 & 0.0 & 0.0 & 82.8 & 17.2 & 442 \\
\hline init_vars & a-2 & 11 & 9 & 0.0 & 0.0 & 0.0 & 82.8 & 17.2 & 442 \\
\hline mapcolor' & $a-0$ & 10 & 13 & 0.0 & 0.0 & 0.0 & 18.1 & 81.9 & 188 \\
\hline mapcolor' & a-1 & 16 & 16 & 0.0 & 0.0 & 0.0 & 14.6 & 85.4 & 261 \\
\hline mapcolor' & a-2 & 19 & 20 & 0.0 & 0.0 & 0.0 & 11.8 & 88.2 & 323 \\
\hline mapcolor & $a-0$ & 4 & 8 & 0.0 & 0.0 & 0.0 & 78.5 & 21.5 & 107 \\
\hline mapcolor & a-1 & 8 & 12 & 0.0 & 0.0 & 0.0 & 80.9 & 19.1 & 173 \\
\hline mapcolor & a-2 & 16 & 19 & 0.0 & 0.0 & 0.0 & 85.4 & 14.6 & 315 \\
\hline grammar & $a-0$ & 0 & 6 & 0.0 & 0.0 & 0.0 & 78.6 & 21.4 & 98 \\
\hline grammar & a-1 & 0 & 6 & 0.0 & 0.0 & 0.0 & 91.0 & 9.0 & 111 \\
\hline grammar & $\mathrm{a}-2$ & 0 & 6 & 0.0 & 0.0 & 0.0 & 91.0 & 9.0 & 111 \\
\hline browse & $a-0$ & 34 & 28 & 0.0 & 0.0 & 0.0 & 49.1 & 50.9 & 636 \\
\hline browse & a-1 & 42 & 31 & 0.0 & 0.0 & 0.0 & 50.1 & 49.9 & 722 \\
\hline browse & $a-2$ & 45 & 35 & 0.0 & 0.0 & 0.0 & 48.0 & 52.0 & 861 \\
\hline browse_u & $a-0$ & 39 & 33 & 0.0 & 0.0 & 0.0 & 49.1 & 50.9 & 709 \\
\hline browse_u & a-1 & 47 & 36 & 0.0 & 0.0 & 0.0 & 49.9 & 50.1 & 799 \\
\hline browse_u & $a-2$ & 50 & 40 & 0.0 & 0.0 & 0.0 & 47.7 & 52.3 & 944 \\
\hline bid & $a-0$ & 7 & 24 & 0.0 & 0.0 & 0.0 & 95.2 & 4.8 & 377 \\
\hline bid & $a-1$ & 15 & 29 & 0.0 & 0.0 & 0.0 & 95.2 & 4.8 & 497 \\
\hline bid & $a-2$ & 22 & 34 & 0.0 & 0.0 & 0.0 & 95.3 & 4.7 & 639 \\
\hline rdtok & $a-0$ & 52 & 39 & 0.0 & 0.0 & 0.0 & 52.8 & 47.2 & 1785 \\
\hline rdtok & a-1 & 63 & 39 & 0.0 & 0.0 & 0.0 & 54.8 & 45.2 & 1891 \\
\hline rdtok & $a-2$ & 65 & 40 & 0.0 & 0.0 & 0.0 & 55.3 & 44.7 & 1911 \\
\hline deriv & $a-0$ & 22 & 31 & 0.0 & 0.0 & 0.0 & 92.9 & 7.1 & 926 \\
\hline deriv & a-1 & 30 & 40 & 0.0 & 0.0 & 0.0 & 93.6 & 6.4 & 1172 \\
\hline deriv & a-2 & 37 & 46 & 0.0 & 0.0 & 0.0 & 93.6 & 6.4 & 1344 \\
\hline kalah & $a-0$ & 31 & 53 & 0.0 & 0.0 & 0.0 & 92.6 & 7.4 & 984 \\
\hline kalah & a-1 & 36 & 54 & 0.0 & 0.0 & 0.0 & 93.8 & 6.2 & 1331 \\
\hline kalah & a-2 & 53 & 61 & 0.0 & 0.0 & 0.0 & 94.7 & 5.3 & 1781 \\
\hline tarjan & $a-0$ & 79 & 73 & 0.0 & 0.0 & 0.0 & 62.3 & 37.7 & 2186 \\
\hline tarjan & a-1 & 102 & 84 & 0.0 & 0.0 & 0.0 & 64.5 & 35.5 & 3159 \\
\hline tarjan & a-2 & 154 & 110 & 0.0 & 0.0 & 0.0 & 74.1 & 25.9 & 5220 \\
\hline read & $a-0$ & 22 & 29 & 0.0 & 0.0 & 0.0 & 88.7 & 11.3 & 1596 \\
\hline read & a-1 & 52 & 45 & 0.0 & 0.0 & 0.0 & 92.3 & 7.7 & 3905 \\
\hline read & a-2 & 86 & 60 & 0.0 & 0.0 & 0.0 & 93.5 & 6.5 & 5803 \\
\hline ch_db_gr & $a-0$ & 28 & 70 & 0.0 & 0.0 & 0.0 & 95.0 & 5.0 & 2072 \\
\hline ch_db_gr & a-1 & 41 & 82 & 0.0 & 0.0 & 0.0 & 96.2 & 3.8 & 2604 \\
\hline ch_db_gr & $\mathrm{a}-2$ & 60 & 93 & 0.0 & 0.0 & 0.0 & 96.6 & 3.4 & 3543 \\
\hline ch_db & $a-0$ & 33 & 76 & 0.0 & 0.0 & 0.0 & 88.5 & 11.5 & 2459 \\
\hline ch_db & a-1 & 46 & 89 & 0.0 & 0.0 & 0.0 & 87.8 & 12.2 & 3001 \\
\hline ch_db & $\mathrm{a}-2$ & 67 & 102 & 0.0 & 0.0 & 0.0 & 89.7 & 10.3 & 4013 \\
\hline peephole & $a-0$ & 56 & 45 & 0.0 & 0.0 & 0.0 & 50.7 & 49.3 & 2437 \\
\hline peephole & a-1 & 63 & 45 & 0.0 & 0.0 & 0.0 & 51.9 & 48.1 & 2642 \\
\hline peephole & $a-2$ & 56 & 45 & 0.0 & 0.0 & 0.0 & 54.4 & 45.6 & 2509 \\
\hline boyer & $a-0$ & 59 & 46 & 0.0 & 0.0 & 0.0 & 22.9 & 77.1 & 1874 \\
\hline icomp & $a-0$ & 141 & 149 & 0.0 & 0.0 & 0.0 & 34.7 & 65.3 & 4858 \\
\hline icomp & a-1 & 179 & 185 & 0.0 & 0.0 & 0.0 & 37.0 & 63.0 & 5987 \\
\hline icomp & a-2 & 220 & 221 & 0.0 & 0.0 & 0.0 & 39.4 & 60.6 & 7194 \\
\hline
\end{tabular}

